\title{
Analytic Continuation of Generating Functions for \\ Two-Dimensional Random Walks in the Quarter Plane
}

\author{
by \\ Simona Sklenar
}

\begin{abstract}
A thesis submitted to the Faculty of Graduate and Postdoctoral Affairs in partial fulfillment of the requirements for the degree of

Master of Science

in

Probability and Statistics
\end{abstract}

Carleton University
Ottawa, Ontario

@ 2015

Simona Sklenar 
Dedications

Leos $\&$ Mack

My Father $\mathcal{G}$ Mother $\mathcal{G}$ Jon

Yiqiang Q. Zhao 


\section{Acknowledgements}

I am forever grateful to my supervisor Dr. Yiqiang Zhao. He is so special and kind and wonderful. He is an amazing teacher. He has taught me so much, he has motivated me, he has helped me, he has supported me, and he always makes me remember my love for math. I am honored to be his student. Thank you Dr. Zhao!

Thank you Joane Emard and Brian Young! You have helped my family and supported us through this entire time.

Thank you Carleton University and The School of Mathematics and Statistics! Thank you to all my professors throughout the years. Thank you Dr. Brian Mortimer, Dr. Barbara Szyszkowicz, Dr. Matthias Neufang, Dr. Shirley Mills and Dr. Michael J. Moore. Thank you Tracie Barkley and Cate Palmer for all your help. The School of Mathematics and Statistics is the best school ever!

Thank you to my father for being my inspiration. Thank you to my mother for being the strongest person I know. Without you, I wouldn't be here. Thank you to my brother Jon.

Thank you to Andy Bowcott, Margie Kardash, and Meredyth Bowcott.

And thank you to my ever loving and supportive husband Mack! I love you forever. 


\section{Abstract}

The amount of tangible real life structures and systems that can be represented as queueing models is substantial, and these systems range in importance and complexity. Queueing theory is instrumental to the survey of the performance of these systems, and therefore, a concrete understanding of queueing theory can provide us with the ability to increase the reliability and efficiency of these systems. One of the most salient properties that we can extract from a stable queueing model is its stationary distributions, which provide vast detail and information regarding the model's long term behavior. In this thesis, we will be considering two-dimensional queueing systems which are equivalent to a random walk in the quarter plane. In an attempt to solve for the stationary distributions, we use a two-variable generating function $\pi(x, y)$, which encodes the joint stationary distribution of the random walk, and we derive a functional equation that will connect $\pi(x, y)$ with the single variable generating functions $\pi_{1}(x)$ and $\pi_{2}(y)$, which encode the two boundary stationary distributions of the random walk. Employing suitable conditions, we are able to reduce the functional equation, known as the fundamental form, to one which only contains both $\pi_{1}(x)$ and $\pi_{2}(y)$. A solid comprehension of $\pi_{1}(x)$ and $\pi_{2}(y)$ can lead us to an understanding of the stationary distributions. However, the assumption here is that all three generating functions are unknown. Since we also assume that the stationary distributions are unknown, we need to find methods in which we can derive meaningful information about the generating functions. Various techniques do exist to compute closed form solutions, or integral representations, for $\pi_{1}(x)$ and $\pi_{2}(y)$, such as using the Riemann-Hilbert boundary value problem. In other instances, solving for $\pi_{1}(x)$ and $\pi_{2}(y)$ is not 
feasible or required. In this case, determining the asymptotic behavior of the generating function around its dominant singularity is the goal and details regarding the asymptotic behavior of the joint and marginal distributions of the random walk are awarded by applying a Tauberian-like theorem. However, in order to successfully employ these methods, the original domains of analyticity for $\pi_{1}(x)$ and $\pi_{2}(y)$ need to be enlarged. Ergo, the analytical continuation of the generating functions $\pi_{1}(x)$ and $\pi_{2}(y)$ is essential.

The process of analytical continuation can become quite intricate. In fact, it has already been shown that both $\pi_{1}(x)$ and $\pi_{2}(y)$ are meromorphic in their respective cut planes. Nonetheless, the proof for this result is also very elaborate and requires a strong knowledge of a wide scope of mathematical topics, including Riemann surfaces, Galois theory and sheaf cohomology. Therefore, the purpose of this thesis is two fold. On the one hand, we would like to provide less complicated material that is accessible to students belonging to diverse fields of study that can benefit from queueing theory but may lack the mathematical skills required to understand other available literature concerning random walks and analytic continuation. On the other hand, if we attempt to analytically continue $\pi_{1}(x)$ and $\pi_{2}(y)$ using rudimentary methods and keeping company with only the complex plane, then perhaps we may discover key information or a consistent result that can be applied to all models, even higher dimensional ones. 


\section{Contents}

Dedications $\quad$ ii

Acknowledgements

Abstract iv

1 Markov Chains 1

1.1 Discrete-Time Markov Chains . . . . . . . . . . . . . . . . . . 2

1.2 Continuous-Time Markov Chains . . . . . . . . . . . . . . . . . 8

1.3 Uniformization . . . . . . . . . . . . . . . . . . . . . 10

1.4 Two-Dimensional Markov Chains . . . . . . . . . . . . . . . . . . 12

2 Random Walks in the Quarter Plane 16

2.1 Random Walks . . . . . . . . . . . . . . . . . . . . . 17

2.2 The Fundamental Form . . . . . . . . . . . . . . . . . . . . . . . . 20

$2.3 \quad$ Ergodicity Conditions . . . . . . . . . . . . . . . . . . . . . . 28

3 Kernel Equation \& Branches 32

3.1 Kernel Equation \& Branch Points . . . . . . . . . . . . . . . . . . 33 
3.2 The Branches of $Y_{ \pm}(x) \& X_{ \pm}(y)$. . . . . . . . . . . . . . . . . . . 40

3.3 The contours $\partial D_{y} \& \partial D_{x}$. . . . . . . . . . . . . . . . . . . 54

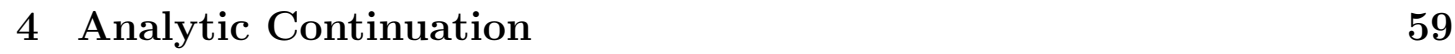

4.1 On the Importance of Analytic Continuation . . . . . . . . . . . . 59

4.2 Power Series \& Analytic Continuation . . . . . . . . . . . . . . . 64

4.3 Two Coupled Processor Model . . . . . . . . . . . . . . . . . . . . 73

4.4 Wireless Three-Hop Networks Model . . . . . . . . . . . . . . . . 86

$\begin{array}{lll}5 & \text { Conclusion and Future Work } & 97\end{array}$

5.1 Conclusion . . . . . . . . . . . . . . . . . . . . . 97

5.2 Future Work . . . . . . . . . . . . . . . . . . . . . . . . . 98

$\begin{array}{ll}\text { Bibliography } & 98\end{array}$ 


\section{Chapter 1}

\section{Markov Chains}

A queue is a waiting line, composed of a variety of objects, including people, vehicles and network packets. Queueing theory is the mathematical study of queues. Agner Krarup Erlang initiated the study of queueing theory when he created models to describe the Copenhagen telephone exchange, which he developed over several years in the first decade of the 20th century [13]. In 1906 Andrey Markov wrote a paper that first described how queues can be reduced to probabilistic objects known as Markov chains [13]. In fact, it has been shown that most queueing models can be interpreted as Markov chains, and, as a consequence, Markov chains are employed as the primary tools for the mathematical analysis involved in queueing theory [13. On that note, we begin with a brief review of Markov chains and present important results that will be applied later on. 


\subsection{Discrete-Time Markov Chains}

A stochastic process $\{X(t): t \in T\}$ is a collection of random variables, defined on a given probability space, such that, for every $t \in T$, where $T \subseteq[0, \infty), X(t)$ is a random variable.

We can further classify the stochastic process based on the cardinality of $T$ : if $T$ is a countable set, then $\{X(t): t \in T\}$ is called a discrete-time process and if $T$ is an interval of the real line, then $\{X(t): t \in T\}$ is called a continuous-time process . Often, the index $t$ is construed to be time, and as such, the random variable $X(t)$ is referred to as the state of the process at time $t$. Furthermore, the set of all possible values that $X(t)$ can assume is called the state space of the process, which can either be countably infinite, including finite, or uncountably infinite. The former is referred to as a discrete-state process, and the latter is called a continuous-state process.

A stochastic process $\{X(t): t \in T\}$ is said to be a Markov process if, for every $t_{1}<\cdots<t_{n}<t_{n+1}$,

$$
\begin{aligned}
& P\left\{X\left(t_{n+1}\right) \leq x_{n+1} \mid X\left(t_{1}\right)=x_{1}, \ldots, X\left(t_{n}\right)=x_{n}\right\} \\
& \quad=P\left\{X\left(t_{n+1}\right) \leq x_{n+1} \mid X\left(t_{n}\right)=x_{n}\right\} .
\end{aligned}
$$

The preceding equation illustrates the defining feature of a Markov process, known as the Markov property or the memoryless property: given the present state, the future state of the process depends only on the present state and not on the past 
history.

Note: From this point on, when we speak of a discrete-time Markov process or a continuous-time Markov process, we will assume that both Markov processes have a discrete state space.

A discrete-state Markov process is called a Markov chain. When we consider a discrete-time Markov chain, then we denote the random variables of the Markov chain as $\left\{X_{n}: n \in \mathbb{N}_{0}\right\}$ and the state space to be $\mathbb{N}_{0}$, where $\mathbb{N}_{0}$ is the set of all non negative integers, that is, the set $\{\mathbb{N} \cup\{0\}\}$. If $X_{n}=i$ then we say that the process is in state $i$ at time $n$. We further characterize the discrete-time Markov chain by the value $p_{i j}$, which is the probability that if the process is currently in state $i$ then the next transition will be to state $j$. More formally, the one-step transition probabilities $p_{i j}$ for $\left\{X_{n}: n \in \mathbb{N}_{0}\right\}$ are defined as:

$$
\begin{aligned}
& P\left\{X_{n+1}=j \mid X_{n}=i, \ldots, X_{0}=i_{0}\right\}=P\left\{X_{n+1}=j \mid X_{n}=i\right\}=p_{i j}, \\
& \text { where } \quad p_{i j} \geq 0 \quad \text { and } \quad \sum_{j=0}^{\infty} p_{i j}=1 \quad \text { for } i \in \mathbb{N}_{0} .
\end{aligned}
$$

We can further define the probability that the process, when in state $i$ will be in state $j$ after $n$ additional transitions, denoted as $p_{i j}^{n}$, for $n, i, j \in \mathbb{N}_{0}$, as:

$$
p_{i j}^{n}=P\left\{X_{n+k}=j \mid X_{k}=i\right\}
$$

When $P\left\{X_{n+1}=j \mid X_{n}=i\right\}$ is independent of $n$ then the Markov chain is said to possess stationary transition probabilities and the process is called a homogenous 
Markov chain, otherwise it is a nonhomogenous Markov chain.

Note: From here on, we shall assume that the discrete-time Markov chain is homogenous.

We now define the one-step transition probability matrix $\mathbf{P}$ of $\left\{X_{n}: n \in \mathbb{N}_{0}\right\}$ as a stochastic matrix whose $(i, j)^{t h}$ entry denotes the probability that the chain's next step, from state $i$, is into state $j$ and the $n$-step transition probability matrix $\mathbf{P}^{(n)}$ of $\left\{X_{n}: n \in \mathbb{N}_{0}\right\}$ as a stochastic matrix whose $(i, j)^{t h}$ entry denotes the probability that the chain will enter state $j$, from state $i$, in $n$ steps. A significant connection between the two matrices is found via the Chapman-Kolmogorov equations, which will not be stated but can be found in any stochastic textbook. These equations demonstrate that $\mathbf{P}^{(n)}=\mathbf{P}^{n}$ 14 and hence, offer a valuable method in deriving the $n$-step transition probabilities: we simply multiply $\mathbf{P}$ by itself $n$ times to obtain $\mathbf{P}^{(n)}$.

The matrix $\mathbf{P}^{(n)}$ clearly provides a lot of information in terms of predicting future habits and patterns of mathematical systems. For example, take weather forecasting. If we are able to model rain in a certain area as a two-state discretetime Markov chain, where the process is in state 0 if it rains and the process is in state 1 if it does not rain, then the value $p_{1,1}^{(12)}$ represents the probability that it will not rain in 12 days, given that it is not raining today. Being able to derive the above probability is very convenient for many reasons. An even stronger mechanism employed for making auguries about a discrete-time Markov chain is to let $\lim _{n \rightarrow \infty} \mathbf{P}^{(n)}$ and determine whether this limit exists and, if so, what the 
limiting values are.

Before we investigate the limiting behavior of a discrete-time Markov chain, we still need some definitions. State $j$ is said to be accessible from state $i$ if there exists an $n \geq 0$ such that $p_{i j}^{n}>0$. Two states $i$ and $j$ are said to communicate if $i$ and $j$ are accessible to each other. Two states belong in the same class if they communicate. If all states in a Markov chain communicate with each other, then there is only one class and the Markov chain is called irreducible. A state $i$ is called recurrent if the probability that, starting in state $i$, the process will reenter state $i$ at some later time, is 1 . Furthermore, if state $i$ is recurrent, then it is considered positive recurrent if, when starting in state $i$, the expected time until the process returns to state $i$ is finite. A state $i$ is said to have period $d$ if $P_{i i}^{n}=0$ whenever $n$ is not divisible by $d$ and $d$ is the largest integer with this property. A state with period 1 is called aperiodic.

Positive recurrent aperiodic states are called ergodic.

We now define $\pi_{j}=\lim _{n \rightarrow \infty} p_{i j}^{n}$. If there exists a probability vector $\pi$, whose $j^{\text {th }}$ coordinate is $\pi_{j}$, such that $\pi \mathbf{P}=\pi$, where $\mathbf{P}$ is the one-step transition probability matrix of a Markov chain, then $\pi$ is called the stationary distribution for the Markov chain. The following important theorem, which we state without proof, tells us the specific properties that a Markov chain needs to possess in order to guarantee the existence and uniqueness of $\pi$. 
Theorem 1.1 [14]: If a discrete-time Markov chain is irreducible and ergodic, then for every $j \in \mathbb{N}_{0}$,

$\pi_{j}=\lim _{n \rightarrow \infty} p_{i j}^{n}$ exists,

and $\pi_{j}$ is the unique non negative solution of

$\pi_{j}=\sum_{i=0}^{\infty} \pi_{i} p_{i j} \quad$ where $\quad \sum_{j=0}^{\infty} \pi_{i}=1$.

We can immediately see the importance of the above theorem. In effect, it is telling us that irrespective of our initial state and irrespective of our initial distribution, if we allow our chain to run for a long time, then the distribution of $X_{n}$ will be the stationary distribution. Moreover, if the initial state is chosen in compliance with the stationary probabilities $\pi_{j}$, then the probability of being in state $j$ at any time $n$ is also $\pi_{j}$. So knowing that a discrete-time Markov chain has the very attractive feature of being both irreducible and ergodic allows us to compute the long run proportion of time that the chain is in at any state. Clearly, the stationary distribution of any system, provided it exists, gives us crucial information when analyzing the behavior of a Markov process and gives us the ability to make strong predictions about system performance. 
Remark: If the discrete-time Markov chain is not aperiodic, then $\lim _{n \rightarrow \infty} p_{i j}^{n}$ may not exist. Consider the easy example where the transition matrix $\mathbf{P}$ of a Markov chain is defined as

$$
\left[\begin{array}{ll}
0 & 1 \\
1 & 0
\end{array}\right]
$$

In this case, $\pi=\left[\frac{1}{2}, \frac{1}{2}\right]$ is the unique solution to $\pi \mathbf{P}=\pi$. However,

$$
\mathbf{P}^{n}= \begin{cases}{\left[\begin{array}{ll}
0 & 1 \\
1 & 0
\end{array}\right],} & \text { if } n=1,3,5, \ldots \\
{\left[\begin{array}{ll}
1 & 0 \\
0 & 1
\end{array}\right],} & \text { if } n=2,4,6, \ldots\end{cases}
$$

and it is clear that $\lim _{n \rightarrow \infty} \mathbf{P}^{n}$ does not exist.

The above example demonstrates the notion that limiting probabilities are not necessarily equal to the stationary probabilities. Although the limit result $\pi_{j}=\lim _{n \rightarrow \infty} p_{i j}^{(n)}$ bears little computational value, it allows us to justify using the stationary distribution as an analysis tool when examining the behavior of real life systems, since most real life systems do not start out stationary. 


\subsection{Continuous-Time Markov Chains}

In accordance with the discrete-time Markov chain, $\{X(t): t \in[0, \infty)\}$ is a continuous-time Markov chain, if for all $s, t \in[0, \infty)$ and states $i, j$ and $x(u)$,

$P\{X(t+s)=j \mid X(s)=i, X(u)=x(u), 0 \leq u<s\}=P\{X(t+s)=j \mid X(s)=i\}$.

When $P\{X(t+s)=j \mid X(s)=i\}$ is independent of $s$, then the continuous-time Markov chain is said to have stationary transition probabilities.

Note: From here on, we shall assume that the continuous-time Markov chain has stationary transition probabilities.

It can be easily shown, using the memoryless property of a Markov process, that if we let $T_{i}$ denote the amount of time that the chain stays in state $i$ before leaving to another state $j$, where $i \neq j$, then $T_{i}$ is exponentially distributed with

mean $\frac{1}{v_{i}}$. Therefore, we can describe a continuous-time Markov chain, as a random process that moves along in the same manner as a discrete-time Markov chain, with a discrete state space, but the amount of time that the continuous-time Markov chain spends in each state, before making a transition to another state, is exponentially distributed. Specifically, for state $i$, the rate at which the process leaves to another state different from state $i$, is $v_{i}$. Furthermore, if we define $p_{i j}$ to be the probability that the process enters state $j$ after it leaves state $i$, then we have, for all $i, p_{i i}=0$ and $\sum_{j} p_{i j}=1$.

Another important quantity of interest is the instantaneous transition rate $q_{i j}$. 
We know that $v_{i}$ is the rate at which the process makes a transition from state $i$ and $p_{i j}$ is the probability that this transition enters state $j$, so $q_{i j}$ is the rate at which the process leaves state $i$ and enters state $j$. We define $q_{i j}$, for any pair of states $i$ and $j$, as $q_{i j}=v_{i} p_{i j}$.

From the definition of $q_{i j}$, we see that

$v_{i}=\sum_{j} q_{i j} \quad$ and $\quad p_{i j}=\frac{q_{i j}}{v_{i}}$.

In order to study the limiting probabilities of a continuous-time Markov chain, we need one more definition, which is similar to the discrete-time version of the probabilities $p_{i j}^{(n)}$, but a smidgen more complicated: the transition probability of the continuous-time Markov chain. The transition probability is the probability that the process, currently in state $i$, will enter state $j$ a time $t$ later, and this quantity is denoted by

$$
p_{i j}(t)=P\{X(t+s)=j \mid X(s)=i\} .
$$

Naturally then, when considering the long term behavior of a continuous-time Markov chain, we analyze the consequences of letting time run off to infinity. If we assume that a limiting value does exist, say $p_{j}$ and is independent of the initial state $i$, then we are looking at: $p_{j}=\lim _{t \rightarrow \infty} p_{i j}(t)$.

To solve these limiting probabilities, we use the following set of equations:

$v_{j} p_{j}=\sum_{i \neq j} q_{j k} p_{k} \quad$ for all states $j \quad$ and $\quad \sum_{j} p_{j}=1$.

The first set of equation $v_{j} p_{j}=\sum_{i \neq j} q_{j k} p_{k}$ is derived from Kolmogorov's forward 
equations [14], which we will not state but can be found in any stochastic textbook. These equations are referred to as a set of balance equations since the left hand side represents the rate at which the process leaves state $j$ and the right hand side represents the rate at which the process enters state $j$.

Parallel to the discrete-time Markov chain case, we still require certain conditions to be met so as to guarantee the existence of the limiting probabilities for the continuous-time Markov chain. In particular, we have the following theorem which will be stated without proof.

Theorem 1.2 [14]: If a continuous-time Markov chain is irreducible and positive recurrent, then

$p_{j} \equiv \lim _{t \rightarrow \infty} p_{i j}(t)$ exists and is independent of $i$.

Note: In contrast to the discrete-time Markov chain, there is no issue of periodicity for continuous-time Markov chain. In this sense, we can interpret $p_{j}$ as both the limiting distribution and as the stationary distribution. When the limiting probabilities $p_{j}$ exist, we call the continuous-time Markov chain ergodic.

\subsection{Uniformization}

Now suppose we have some ergodic continuous-time Markov chain $\{X(t): t \in$ $[0, \infty)\}$ with parameters $v_{i}$. Let $Y_{n}$ be the state of $X(t)$ after $n$ transitions. Then $\left\{Y_{n}: n \in \mathbb{N}_{0}\right\}$ is a discrete-time Markov chain with transition probabilities $p_{i j}=$ 
$\frac{q_{i j}}{v_{i}}$ and we call it an embedded chain [14].

Remark: When $v_{i} \equiv v$ then $\pi_{j}=p_{j}$.

The preceding remark demonstrates that perhaps it is possible to extract a discrete-time Markov chain from a continuous one, where the stationary distributions remain intact. And indeed there is, by way of uniformization. The idea behind uniformization takes a continuous-time Markov chain, with varying bounded $v_{i} \mathrm{~s}$, and chooses a uniform parameter $v$ such that, for all states $i, v \geq v_{i}$ [14]. To compensate, a fictitious transition from a state to itself is added and the new embedded Markov chain is defined as follows: If the process is in state $i$, then the amount of time it stays in state $i$ is exponentially distributed with mean $\frac{1}{v}$ and then the process will leave state $i$ with probability $\frac{v_{i}}{v}$ or return to state $i$ with probability $1-\frac{v_{i}}{v}$. That is, our new discrete-time Markov chain has transition probabilities defined as:

$p_{i i}^{*}=1-\frac{v_{i}}{v} \quad$ and $\quad p_{i j}^{*}=\frac{v_{i}}{v} p_{i j} \quad$ for all $i \neq j[14$.

Recalling the definition of a Poisson process, in the same vein as this introduction, it is a process whose state space is $\mathbb{N}_{0}$, the amount of time it spends in each state $i$ is exponentially distributed with mean $\frac{1}{\lambda}$, so that $v_{i}=\lambda$ for every $i$, and, $p_{i j}=1$ if $j=i+1$ and 0 otherwise. We can see then, that our new embedded Markov chain has jumps which occur according to a Poisson process with rate $v$. In this way, uniformization allows us to view almost all continuous-time Markov chains, with parameters $v_{i}$ and $p_{i j}$, as an amalgamation of a Poisson process with 
rate $v$ and a discrete-time Markov chain with transition probabilities $p_{i j}^{*}$.

One of the most powerful results of uniformization which we will exploit later on, is that the uniformized Markov chain has the same stationary distribution as the original continuous-time Markov chain [14]. It is for this very reason that the use of uniformized Markov chains to study the properties of continuous-time Markov chains constitutes a very significant method in applied probability theory.

\subsection{Two-Dimensional Markov Chains}

Let us briefly backtrack to the continuous-time Markov chain and look at birth and death queueing models, which are exponential queueing systems where the arrival and departure rates depend on the number of people, say, in the system. If there are $n$ customers in the system, then the time until the next arrival is exponential with rate $\lambda_{n}$ and is independent of the time of the next departure, which is also exponential with rate $\mu_{n}$. Moreover, the time until either the next arrival or departure occurs is also exponentially distributed with rate $\lambda_{n}+\mu_{n}$ [14].

One of the most easily understood examples of a one-dimensional queueing system, that is, a system with only one queue, is the $M / M / 1$ system. This is modeled as the birth and death process described above, where, if we let $\{X(t)$ : $t \geq 0\}$ denote the number in the system at time $t$, then

$$
\mu_{n}=\mu \quad \text { for } n \geq 1 \quad \text { and } \quad \lambda_{n}=\lambda \text { for } n \geq 0 .
$$

We can visualize the $M / M / 1$ system as people arriving to a single-server service 
system, in accordance with a Poisson process having rate $\lambda$, so that the times between successive arrivals are independent exponential random variables having mean $\frac{1}{\lambda}$. Upon arrival, the customer either goes directly to the server, if the server is free, or joins a queue, that is, a line or a room, where the customer waits. We assume this queue has infinite capacity. When the server is finished with the customer, the customer leaves the system and if there is another customer in line, then that new customer enters service. The successive service times are also assumed to be independent exponential random variables having mean $\frac{1}{\mu}$. Indeed, the two $M \mathrm{~s}$, in the name $M / M / 1$ refers to the fact that both the interarrival time and service time are exponentially distributed, and hence Markovian [14. The 1 refers to the fact that there is only one server.

To determine the limiting probabilities, we use the balance equations and get the following relation: $\lambda P_{n}=\mu P_{n+1}$ for all $n \geq 0$. Furthermore, using the fact that $\sum_{n=0}^{\infty} P_{n}=1$, we obtain:

$$
P_{0}=\frac{1}{1+\sum_{n=1}^{\infty}\left(\frac{\lambda}{\mu}\right)^{n}}
$$

which gives:

$$
P_{n}=\frac{\left(\frac{\lambda}{\mu}\right)^{n}}{1+\sum_{n=1}^{\infty}\left(\frac{\lambda}{\mu}\right)^{n}}=\left(\frac{\lambda}{\mu}\right)^{n}\left(1-\frac{\lambda}{\mu}\right) \quad \text { for } \quad \frac{\lambda}{\mu}<1 \quad \text { and } \quad n \geq 0 .
$$

Even without computing $P_{n}$, we can intuitively see that for this system to be stable, we need $\lambda<\mu$. If $\lambda>\mu$, then the customers would arrive at a faster rate 
than they could be served and the queue would blow up, so to speak.

Of course there are various types of one-dimensional queueing systems, with varying degrees of distributions, amount of servers, the way in which the customers are served, and the amount of customers the queue can hold. But the common feature amongst all these models is that they only have one queue. We are interested in a queueing system that has two queues, $\mathbf{Q}(\mathbf{t})=\left(Q_{1}(t), Q_{2}(t)\right)$, which can be modeled as birth and death processes. One of the easiest examples of a two-dimensional queueing system is that of a tandem queue. This is described as a two-server service system in which customers arrive at a Poisson rate $\lambda$ to server 1. If the server is a busy with a current customer, the arriving customers waits in a queue. Otherwise, the arriving customer goes directly to server 1 . The service time of server 1 is exponentially distributed with mean $\frac{1}{\mu_{1}}$. After being served by server 1 , the customer goes to server 2. Again, if server 2 is busy, the customer waits in a second queue, otherwise the customer goes directly to server 2 . The service time of server 2 is also exponentially distributed with mean $\frac{1}{\mu_{2}}$. Once server 2 has served the customer, the customer leaves the system. We also assume that both queues have infinity capacity and each server serves one customer at a time. We can see that server 1 is faced with an $M / M / 1$ queue and server 2 is faced with an $M / M / 1$ queue.

The computational aspect of determining the limiting probabilities $p_{m, n}$ from the 
balance equations can be quite involved. However, it has been shown that [14]:

$$
p_{m, n}=\left(\frac{\lambda}{\mu_{1}}\right)^{m}\left(1-\frac{\lambda}{\mu_{1}}\right)\left(\frac{\lambda}{\mu_{2}}\right)^{n}\left(1-\frac{\lambda}{\mu_{2}}\right) .
$$

Intuitively, we see that the system is stable $\lambda<\mu_{1}$ and $\lambda<\mu_{2}$. 


\section{Chapter 2}

\section{Random Walks in the Quarter}

\section{Plane}

There is a myriad of interesting two-dimensional queueing systems, including tandem queues with coupled processor, where one idle server helps the other, a Jackson network, which has two arrival rates and preemptive priority queueing systems, where the arriving customers are placed into two classes, higher and lower priority. When the queues in two-dimensional system represent continuous-time Markov chains and have bounded rates, then we know from the previous section that we can convert these chains into discrete-time ones, through uniformization. This chapter will focus on how we can model a two-dimensional system as a random walk in the quarter plane and then we will introduce a functional equation that joins the probability generating functions for the joint stationary distribution $\pi_{m, n}$, of the random walk, with the two generating functions for the boundary 
stationary distributions, $\pi_{m, 0}$ and $\pi_{0, n}$, of the random walk.

\subsection{Random Walks}

The random walk in the quarter plane that we will consider is one that can be classically described as a drunk walking around in an area with walls (boundaries) in two adjacent sides which are both infinite in length. Visually, the construct of the area and two walls is identical to the first quadrant of the coordinate plane. If the drunk is in the interior of the two boundaries, then he can move to one of the eight positions available to him, or stay in the same position. The probability that he moves to one of these new positions, or stays put, is denoted by $p_{i, j}$ for $i, j=0, \pm 1$. If the drunk is on the horizontal wall, excluding the point $(0,0)$, then he has five available positions to choose from for his next step, which will either reflect off the boundary, move left or right on the boundary, or remain in the same position, with probability $p_{i, j}^{(1)}$ for $i=0, \pm 1$ and $j=0,1$. Similarly, on the vertical boundary, excluding the point $(0,0)$, the drunk also only has five choices for his next step, which will also be a reflection off the boundary, a movement up or down on the boundary, or no movement, with probability $p_{i, j}^{(2)}$ for $i=0,1$ and $j=0, \pm 1$. Finally, at the point $(0,0)$, the drunk can either move up the vertical boundary, move right on the horizontal boundary, move into the one position available to him in the interior, or stay in the same position, with probability $p_{i, j}^{(0)}$ for $i, j=0,1$. Note that we have assumed that the random walk is homogeneous and that the steps are bounded by one. The latter implies that the drunk is unable to hop 
over one of his available choices, into another position. He must either stay put or make one step to one of the neighboring positions. Finally, we assume that $p_{0,0}<1$ since otherwise the random walk would be degenerative: the drunk would be too inebriated to leave his initial position [17].

If we define the random variables $\mathbf{X}_{+}, \mathbf{X}_{1}, \mathbf{X}_{2}$ and $\mathbf{X}_{0}$ to have the distributions

$$
\mathbf{X}_{+} \sim p_{i, j}, \quad \mathbf{X}_{1} \sim p_{i, j}^{(1)}, \quad \mathbf{X}_{2} \sim p_{i, j}^{(2)} \quad \text { and } \quad \mathbf{X}_{0} \sim p_{i, j}^{(0)}
$$

then the random walk in the quarter plane, $\mathbf{L}_{t}=\left(L_{1}(t), L_{2}(t)\right)$, is a two-dimensional discrete-time Markov chain [17].

We define the union of the following 4 sets as the state space of $\mathbf{L}_{t}$ :

$$
\begin{aligned}
& S_{+}=\{(m, n): m, n \in \mathbb{N} \backslash\{0\}\} \text { as the interior of quarter plane, } \\
& S^{(1)}=\{(m, 0): m \in \mathbb{N} \backslash\{0\}\} \text { as the horizontal axis, } \\
& S^{(2)}=\{(0, n): n \in \mathbb{N} \backslash\{0\}\} \text { as the vertical axis and } \\
& S^{(0)} \text { as the origin }(0,0)
\end{aligned}
$$

Moreover, the random walk $\mathbf{L}_{t}$ has transition probabilities, for $k=0,1,2$,

$$
\begin{aligned}
& P\left(\mathbf{L}_{t+1}=\left(m_{2}, n_{2}\right) \mid \mathbf{L}_{t}=\left(m_{1}, n_{1}\right)\right) \\
& = \begin{cases}P\left(\mathbf{X}_{+}=\left(m_{2}-m_{1}, n_{2}-n_{1}\right)\right. & \text { if }\left(m_{2}, n_{2}\right) \in \mathbb{N}_{0}^{2},\left(m_{1}, n_{1}\right) \in S_{+} \\
P\left(\mathbf{X}_{k}=\left(m_{2}-m_{1}, n_{2}-n_{1}\right)\right. & \text { if }\left(m_{2}, n_{2}\right) \in \mathbb{N}_{0}^{2},\left(m_{1}, n_{1}\right) \in S^{(k)}\end{cases}
\end{aligned}
$$

If we let $\mathbf{P}$ denote the transition probability matrix and $\pi=\left(\pi_{i, j}\right)$ be the unknown 
stationary equations to $\pi \mathbf{P}=\pi$, then $\pi \mathbf{P}=\pi$ is equivalent to the following systems of equations [17]:

$$
\begin{aligned}
& \left(1-p_{0,0}^{(0)}\right) \pi_{0,0}=p_{-1,0}^{(1)} \pi_{1,0}+p_{0,-1}^{(2)} \pi_{0,1}+p_{-1,-1} \pi_{1,1} \\
& \left(1-p_{0,0}^{(1)}\right) \pi_{1,0}=p_{1,0}^{(0)} \pi_{0,0}+p_{-1,0}^{(1)} \pi_{2,0}+p_{-1,-1} \pi_{2,1}+p_{1,-1}^{(2)} \pi_{0,1}+p_{0,-1} \pi_{1,1} \\
& \left(1-p_{0,0}^{(2)}\right) \pi_{0,1}=p_{0,1}^{(0)} \pi_{0,0}+p_{0,-1}^{(1)} \pi_{0,2}+p_{-1,-1} \pi_{1,2}+p_{-1,1}^{(1)} \pi_{1,0}+p_{-1,0} \pi_{1,1}
\end{aligned}
$$

For $i \geq 2$,

$$
\left(1-p_{0,0}^{(1)}\right) \pi_{i, 0}=p_{1,0}^{(1)} \pi_{i-1,0}+p_{-1,0}^{(1)} \pi_{i+1,0}+p_{-1,-1} \pi_{i+1,1}+p_{1,-1} \pi_{i-1,1}+p_{0,-1} \pi_{i, 1}
$$

for $j \geq 2$,

$$
\left(1-p_{0,0}^{(2)}\right) \pi_{0, j}=p_{0,1}^{(2)} \pi_{0, j-1}+p_{0,-1}^{(2)} \pi_{0, j+1}+p_{-1,-1} \pi_{1, j+1}+p_{-1,1} \pi_{1, j-1}+p_{-1,0} \pi_{1, j}
$$

and, for both $i \geq 2$ and $j \geq 2$,

$$
\begin{aligned}
\left(1-p_{0,0}\right) \pi_{i, j} & =p_{1,-1} \pi_{i-1, j+1}+p_{-1,-1} \pi_{i+1, j+1}+p_{0,-1} \pi_{i, j+1}+p_{1,0} \pi_{i-1, j}+p_{-1,0} \pi_{i+1, j} \\
& +p_{1,1} \pi_{i-1, j-1}+p_{0,1} \pi_{i, j-1}+p_{-1,1} \pi_{i+1, j-1}+p .
\end{aligned}
$$

Note: In terms of notation, since we are interested in two-dimensional queueing system that can be modeled as the random walk in the quarter plane described above, we denote the horizontal axis as $Q_{1}$ and it represents the number of customers, say, in the first queue. Similarly, we denote the vertical axis as $Q_{2}$ and it represents the number of customers in the second queue. 


\subsection{The Fundamental Form}

The generating function associated with a sequence $\left\{a_{n}: n \in \mathbb{N}_{0}\right\}$ is a power series given by $f(z)=\sum_{n=0}^{\infty} a_{n} z^{n}$ for $z \in \mathbb{C}$.

A discrete random variable $X$, taking on non-negative integers, with probability mass function $P(X=n)=p_{n}$ is fully specified by the sequence $\left\{p_{n}: n \in \mathbb{N}_{0}\right\}$ and the corresponding generating function

$$
A(z)=\mathbb{E}\left(z^{X}\right)=\sum_{n=0}^{\infty} p_{n} z^{n}, \quad z \in \mathbb{C},
$$

is called a probability generating function. Notice that $0 \leq p_{n} \leq 1$ and $A(1)=1$, so that $A(z)$ is well defined for all $|z| \leq 1$ and converges absolutely for all $|z| \leq 1$ [8]. We will explore the notions of convergence and analyticity for probability generating functions in Chapter 4.

In the multivariate case, if $X=\left(X_{1}, \ldots, X_{d}\right)$ is a discrete random variable taking values in the $d$-dimensional non-negative integer lattice $\{0,1, \ldots\}^{d}$, then, for $z=$ $\left(z_{1}, \ldots, z_{d}\right) \in \mathbb{C}^{d}$, the probability generating function of $X$ is defined as:

$$
A(z)=A\left(z_{1}, \ldots, z_{d}\right)=\mathbb{E}\left(z_{1}^{X_{1}} \cdots z_{d}^{X_{d}}\right)=\sum_{x_{1}=0}^{\infty} \cdots \sum_{x_{d}=0}^{\infty} p\left(x_{1}, \ldots, x_{d}\right) z_{1}^{x_{1}} \cdots z_{d}^{x_{d}} .
$$

The above sum converges absolutely for at least all $z=\left(z_{1}, \ldots, z_{d}\right) \in \mathbb{C}^{d}$ with $\max \left\{\left|z_{1}\right|, \ldots,\left|z_{d}\right|\right\} \leq 1$.

Note: Since we will only be dealing with discrete random variables taking on non-negative integer values, we shall henceforth refer to a probability generating 
function simply as a generating function.

\section{Examples:}

Let $\varrho=\frac{\lambda}{\mu}$. Then, for the $M \backslash M \backslash 1$ queue, with $z \in \mathbb{C}$, we have:

$$
A(z)=\sum_{n=0}^{\infty} p_{n} z^{n}=\sum_{n=0}^{\infty}(\varrho)^{n}(1-\varrho) z^{n}=\frac{1-\varrho}{1-\varrho z} \quad \text { for }|z|<\frac{1}{\varrho} .
$$

For the tandem queue, we let $\varrho_{1}=\frac{\lambda}{\mu_{1}}$ and $\varrho_{2}=\frac{\lambda}{\mu_{2}}$. Then, for $x \in \mathbb{C}_{x}$ and $y \in \mathbb{C}_{y}$, we have

$$
\begin{aligned}
A(x, y) & =\sum_{m=0}^{\infty} \sum_{n=0}^{\infty} p_{m, n} x^{m} y^{n} \\
& =\sum_{x=0}^{\infty} \sum_{y=0}^{\infty}=\left(\varrho_{1}\right)^{m}(1-\varrho)\left(\varrho_{2}\right)^{n}\left(1-\varrho_{2}\right) x^{m} y^{n} \\
& =\frac{1-\varrho_{1}}{1-\varrho_{1} x} \cdot \frac{1-\varrho_{2}}{1-\varrho_{2} y} \quad \text { for }|x|<\frac{1}{\varrho_{1}} \text { and }|y|<\frac{1}{\varrho_{2}} .
\end{aligned}
$$

In a sense, the probability distribution is encoded in its generating function and we see that characterizing $p_{n}$ is equivalent to characterizing $A(z)$ [17]. It is for this reason that generating functions are considered extremely useful tools for analysis, since they offer a power series which can potentially be expressed in a simplified form. In the above example, we were able to derive the generating functions for both queueing systems because we already had prior knowledge as to what $p_{n}$ and $p_{m, n}$ were. Suppose we did not have an expression for $p_{n}$ and $p_{m, n}$ but did have closed form expressions of their generating functions. Then we can work 
our way back, using the expressions and definition of the generating functions, to find $p_{n}$ and $p_{m, n}$. Even if we are unable to solve for $p_{n}$ and $p_{m, n}$ directly, we can still extract valuable properties about these probability distributions from their generating functions. Naturally then, a laconic method in obtaining information about the stationary distributions, $\pi_{m, n}, \pi_{m, 0}$ and $\pi_{0, n}$, assuming they exist, is to study the generating functions $\pi(x, y), \pi_{1}(x)$ and $\pi_{2}(y)$, which are defined as [16]:

$$
\begin{aligned}
& \pi(x, y)=\sum_{m=1}^{\infty} \sum_{n=1}^{\infty} \pi_{m, n} x^{m-1} y^{n-1} \quad \text { for }(x, y) \in \mathbb{C}^{2} \\
& \pi_{1}(x)=\sum_{m=1}^{\infty} \pi_{m, 0} x^{m-1} \quad \text { for } x \in \mathbb{C}_{x} \\
& \pi_{2}(y)=\sum_{n=1}^{\infty} \pi_{0, n} y^{n-1} \quad \text { for } y \in \mathbb{C}_{y}
\end{aligned}
$$

Note: $\pi_{0,0}$ is a constant.

Remark: $\mathbb{C}_{x} \equiv \mathbb{C}$ and $\mathbb{C}_{y} \equiv \mathbb{C}$. The standard notation for a complex number is $z=x+i y$ where $x, y \in \mathbb{R}$. However, in our case, both $x$ and $y$ are complex numbers themselves, in the sense that $x=u+i v$ and $y=a+b i$, where $u, v, a, b \in \mathbb{R}$. It is for this reason that we index the complex plane with either $x$ or $y$ so that we remember that both $x$ and $y$ are complex numbers, living in their respective complex plane. 
Clearly, none of these generating functions are finite for every $x \in \mathbb{C}_{x}$ and $y \in \mathbb{C}_{y}$. However, here is what we do know:

1. $\pi(x, y)$ is analytic in the open domain $\left\{(x, y) \in \mathbb{C}^{2}:|x|<1,|y|<1\right\}$.

2. $\pi(x, y)$ is continuous in the closed domain $\left\{(x, y) \in \mathbb{C}^{2}:|x| \leq 1,|y| \leq 1\right\}$.

Similar results hold for both $\pi_{1}(x)$ and $\pi_{2}(y)$ : both are analytic in the unit disk, in their respective complex planes, and both are continuous on the unit circle, in their respective complex planes. These claims will be proven in Chapter 4 .

We want to find a functional equation between all three unknown generating functions. Indeed, such a functional equation exists, known as the fundamental form. However, we first need to define the remaining ingredients. Accordingly, for $x \in \mathbb{C}_{x}$ and $y \in \mathbb{C}_{y}$, let $[16]$ :

$$
\begin{aligned}
h(x, y) & =x y\left(\sum_{i=-1}^{1} \sum_{j=-1}^{1} p_{i, j} x^{i} y^{j}-1\right) \\
& =a(x) y^{2}+b(x) y+c(x)=\tilde{a}(y) x^{2}+\tilde{b}(y) x+\tilde{c}(y), \\
h_{1}(x, y) & =x\left(\sum_{i=-1}^{1} \sum_{j=0}^{1} p_{i, j}^{(1)} x^{i} y^{j}-1\right) \\
& =a_{1}(x) y+b_{1}(x)=\tilde{a}_{1}(y) x^{2}+\tilde{b}_{1}(y) x+\tilde{c}_{1}(y),
\end{aligned}
$$




$$
\begin{aligned}
h_{2}(x, y) & =y\left(\sum_{i=0}^{1} \sum_{j=-1}^{1} p_{i, j}^{(2)} x^{i} y^{j}-1\right) \\
& =a_{2}(x) y^{2}+b_{2}(x) y+c_{2}(x)=\tilde{a}_{2}(y) x+\tilde{b}_{2}(y), \\
h_{0}(x, y) & =\left(\sum_{i=0}^{1} \sum_{j=0}^{1} p_{i, j}^{(0)} x^{i} y^{j}-1\right) \\
& =a_{0}(x) y+b_{0}(x)=\tilde{a}_{0}(y) x+\tilde{b}_{0}(y),
\end{aligned}
$$

where,

$$
\begin{aligned}
& a(x)=p_{-1,1}+p_{0,1} x+p_{1,1} x^{2} \\
& b(x)=p_{-1,0}-\left(1-p_{0,0}\right) x+p_{1,0} x^{2} \\
& c(x)=p_{-1,-1}+p_{0,-1} x+p_{1,-1} x^{2} \\
& a_{1}(x)=p_{-1,1}^{(1)}+p_{0,1}^{(1)} x+p_{1,1}^{(1)} x^{2}
\end{aligned}
$$




$$
\begin{aligned}
& b_{1}(x)=p_{-1,0}^{(1)}-\left(1-p_{0,0}^{(1)}\right) x+p_{1,0}^{(1)} x^{2} \\
& a_{2}(x)=p_{0,1}^{(2)}+p_{1,1}^{(2)} x \\
& b_{2}(x)=p_{0,0}^{(2)}-1+p_{1,0}^{(2)} x \\
& c_{2}(x)=p_{0,-1}^{(2)}+p_{1,-1}^{(2)} x \\
& \tilde{c}(y)=p_{-1,-1}+p_{-1,0} y+p_{-1,1} y^{2} \\
& \tilde{b}(y)=p_{0,-1}-\left(1-p_{0,0}\right) y+p_{0,1} y^{2} \\
& a_{0}(x)=p_{0,1}^{(0)}+p_{1,1}^{(0)} x \\
& b_{0}(x)=p_{1,0}^{(0)} x-\left(1-p_{0,0}^{(0)}\right) \\
& \tilde{a}(y)=p_{1,-1}+p_{1,0} y+p_{1,1} y^{2} \\
& { }^{2}(x)
\end{aligned}
$$




$$
\begin{aligned}
& \tilde{a}_{0}(y)=p_{1,0}^{(0)}+p_{1,1}^{(0)} y \\
& \tilde{b}_{0}(y)=p_{0,1}^{(0)} y-\left(1-p_{0,0}^{(0)}\right) \\
& \tilde{a}_{1}(y)=p_{1,0}^{(1)}+p_{1,1}^{(1)} y \\
& \tilde{b}_{1}(y)=p_{0,0}^{(1)}-1+P_{0,1}^{(1)} y \\
& \tilde{c}_{1}(y)=p_{-1,0}^{(1)}+p_{-1,1}^{(1)} y \\
& \tilde{a}_{2}(y)=p_{1,-1}^{(2)}+p_{1,0}^{(2)} y+p_{1,1}^{(2)} y^{2} \\
& \tilde{b}_{2}(y)=p_{0,-1}^{(2)}-\left(1-p_{0,0}^{(2)}\right) y+p_{0,1}^{(2)} y^{2} .
\end{aligned}
$$

Now we can state the fundamental form of the random walk, which is given by [16]:

$$
-h(x, y) \pi(x, y)=h_{1}(x, y) \pi_{1}(x)+h_{2}(x, y) \pi_{2}(y)+h_{0}(x, y) \pi_{0,0}
$$

For simplification, due to extensive computation, a proof of the fundamental form 
is not given, although one is provided in (1.3.6) in [2]. The reason why the above functional equation is referred to as fundamental is primarily based on the fact that, through analysis of the functional equation, we can determine, or express, the unknown generating functions. [17]

Often times, authors work with generating functions where the summation begins at 0 , rather than 1 [16]:

$\Pi(x, y)=\sum_{i=0}^{\infty} \sum_{j=0}^{\infty} \pi_{i, j} x^{i} y^{j}, \quad \Pi_{1}(x)=\sum_{i=0}^{\infty} \pi_{i, 0} x^{i}, \quad$ and $\quad \Pi_{2}(y)=\sum_{j=0}^{\infty} \pi_{0, j} y^{j}$.

Then the fundamental form (2.1) becomes:

$$
H(x, y) \Pi(x, y)=H_{1}(x, y) \Pi_{1}(x)+H_{2}(x, y) \Pi_{2}(y)+H_{0}(x, y) \pi_{0,0},
$$

where,

$$
\begin{aligned}
& H(x, y)=-h(x, y) \\
& H_{1}(x, y)=-h(x, y)+h_{1}(x, y) y \\
& H_{2}(x, y)=-h(x, y)+h_{2}(x, y) x \\
& H_{0}(x, y)=h_{0}(x, y) x y+h(x, y)-h_{1}(x, y) y-h_{2}(x, y) x .
\end{aligned}
$$

We will be using the fundamental form (2.1) throughout the rest of our work, although we will employ (2.2) for the Wireless Three-Hop Networks model in Chapter 4. Again, the fundamental form is an exceptionally significant functional equation since it links all of the unknown generating functions together, and, as such, we can cleverly manipulate (2.1) so at to compute or derive an expression 
for the generating functions. However, before we continue our analysis of (2.1), it would be wise to explore when, and under what circumstances, the stationary distributions of the random walk exist.

\subsection{Ergodicity Conditions}

Recall in section 1.1 we showed that a discrete-time Markov chain was ergodic (irreducible, aperiodic, and positive recurrent) if and only if the stationary equations $\pi \mathbf{P}=\pi$ existed and had a unique solution such that $\sum_{\alpha} \pi_{\alpha}=1$. We conclude this chapter by a theorem that gives necessary and sufficient conditions for the random walk to be ergodic. Let:

$$
\begin{aligned}
& \mathcal{D}_{z}(0,1)=\{z \in \mathbb{C}:|z|<1\} \text { be the open unit disk in the } z \text { plane, } \\
& \overline{\mathcal{D}}_{z}(0,1)=\{z \in \mathbb{C}:|z| \leq 1\} \text { be the closed unit disk in the } z \text { plane, } \\
& \partial \mathcal{D}_{z}(0,1)=\{z \in \mathbb{C}:|z|=1\} \text { be the unit circle in the } z \text { plane. }
\end{aligned}
$$

Theorem 2.1 [2]: For the irreducible aperiodic random walk to be ergodic, it is necessary and sufficient that there exists functions $\pi(x, y), \pi_{1}(x)$ and $\pi_{2}(y)$ analytic in $\mathcal{D}_{x}(0,1) \times \mathcal{D}_{y}(0,1), \mathcal{D}_{x}(0,1)$ and $\mathcal{D}_{y}(0,1)$, respectively, and a constant $\pi_{0,0}$, satisfying the fundamental form (2.1), together with the condition that

$$
\sum_{m=0}^{\infty} \sum_{n=0}^{\infty}\left|\pi_{m, n}\right|<\infty .
$$


Proof: The proof follows directly from the sufficient and necessary conditions of an ergodic discrete-time Markov chain, as recalled in the first paragraph of this section.

Note: From this point forward, when we do our analysis on any random walk in the quarter plane, we shall assume that the random walk is ergodic.

Remark: A complex valued function $f(z)$ is called analytic on an open set, if it is differentiable at every point in the open set [12]. We will further explore the idea of analyticity in Chapter 4.

Theorem 2.1 has merit from a theoretical standpoint, but in practice it is not convenient, since the determination of the unknown functions $\pi_{k}$ is required. Instead, to acquire the stability conditions, we turn to a very useful result using mean drifts.

Let us denote the expected one-step transition along the $x$ direction from an interior state as $M_{x}$, the expected one-step transition along the $y$ direction from an interior state as $M_{y}$, and the expected one-step transition along the $x$ direction, or $y$ direction, from a boundary state as $M_{x}^{(k)}$ and $M_{y}^{(k)}$, respectively, for $k=1,2$. 
Then these mean drifts are defined as followed [16]:

$$
\begin{aligned}
& \mathbf{M}=\left(M_{x}, M_{y}\right)=\left(\sum_{i} i\left(\sum_{j} p_{i, j}\right), \sum_{j} j\left(\sum_{i} p_{i, j}\right)\right), \\
& \mathbf{M}^{(1)}=\left(M_{x}^{(1)}, M_{y}^{(1)}\right)=\left(\sum_{i} i\left(\sum_{j} p_{i, j}^{(1)}\right), \sum_{j} j\left(\sum_{i} p_{i, j}^{(1)}\right)\right), \\
& \mathbf{M}^{(2)}=\left(M_{x}^{(2)}, M_{y}^{(2)}\right)=\left(\sum_{i} i\left(\sum_{j} p_{i, j}^{(2)}\right), \sum_{j} j\left(\sum_{i} p_{i, j}^{(2)}\right)\right) .
\end{aligned}
$$

Furthermore, by simple algebra, we can show that:

$$
\begin{aligned}
& M_{x}=\tilde{a}(1)-\tilde{c}(1)=\left.\frac{d}{d x} a(x)\right|_{x=1}+\left.\frac{d}{d x} b(x)\right|_{x=1}+\left.\frac{d}{d x} c(x)\right|_{x=1}, \\
& M_{x}^{(1)}=\tilde{a}_{1}(1)-\tilde{c}_{1}(1)=\left.\frac{d}{d x} a_{1}(x)\right|_{x=1}+\left.\frac{d}{d x} b_{1}(x)\right|_{x=1}, \\
& M_{x}^{(1)}=\tilde{a}_{2}(1)=\left.\frac{d}{d x} a_{2}(x)\right|_{x=1}+\left.\frac{d}{d x} b_{2}(x)\right|_{x=1}+\left.\frac{d}{d x} c_{2}(x)\right|_{x=1}, \\
& M_{y}=a(1)-c(1)=\left.\frac{d}{d y} \tilde{a}(y)\right|_{y=1}+\left.\frac{d}{d y} \tilde{b}(y)\right|_{y=1}+\left.\frac{d}{d y} \tilde{c}(y)\right|_{y=1}, \\
& M_{y}^{(1)}=a_{1}(1)=\left.\frac{d}{d y} \tilde{a}_{1}(y)\right|_{y=1}+\left.\frac{d}{d y} \tilde{b}_{1}(y)\right|_{y=1}+\left.\frac{d}{d y} \tilde{c}_{1}(y)\right|_{y=1}, \\
& M_{y}^{(2)}=a_{2}(1)-c_{2}(1)=\left.\frac{d}{d y} \tilde{a}_{2}(y)\right|_{y=1}+\left.\frac{d}{d y} \tilde{b}_{2}(y)\right|_{y=1} .
\end{aligned}
$$

We use these mean drifts to determine ergodicity conditions for the random walk via Theorem 3.3.1 of [2], which was later amended by Kobayashi and Miyazawa, and will be stated without proof [16]:

Theorem 2.2: When $\boldsymbol{M} \neq 0$, the random walk is ergodic if and only if one of the following three conditions hold:

1. $M_{x}<0, M_{y}<0, M_{x} M_{y}^{(1)}-M_{y} M_{x}^{(1)}<0$ and $M_{y} M_{x}^{(2)}-M_{x} M_{y}^{(2)}<0$;

2. $M_{x}<0, M_{y} \geq 0, M_{y} M_{x}^{(2)}-M_{x} M_{y}^{(2)}<0$ and $M_{x}^{(1)}<0$ if $M_{x}^{(1)}=0$; 
3. $M_{x} \geq 0, M_{y}<0, M_{x} M_{y}^{(1)}-M_{y} M_{x}^{(1)}<0$ and $M_{y}^{(2)}<0$ if $M_{x}^{(2)}=0$.

When the random walk is stable and $\mathbf{M}=0$, then we call the stationary distributions $\pi_{m, 0}$ and $\pi_{0, n}$ heavy-tailed. On the other hand, when $\mathbf{M} \neq 0$, then $\pi_{m, 0}$ and $\pi_{0, n}$ are referred to as light-tailed. Heavy-tailed cases are much more complicated than light-tailed cases and there still remains many open questions concerning the former. In particular, for the heavy-tailed case, it has been shown that $\pi_{1}(x)$ and $\pi_{2}(y)$ cannot be analytically continued past the unit disk, in their respective domains [2]. However, for the light-tailed case, it has been shown that $\pi_{1}(x)$ and $\pi_{2}(y)$ can be analytically continued past the unit disk, in their respective domains [2]. Since the purpose of this thesis is to analytically continue $\pi_{1}(x)$ and $\pi_{2}(y)$ past the unit disk, we will assume that for any random walk we consider, $\mathbf{M} \neq 0$, since otherwise analytic continuation is not feasible.

Note: Notice that the condition $\mathbf{M} \neq 0$ is equivalent to $\tilde{a}(1) \neq \tilde{c}(1)$ and $a(1) \neq$ $c(1)$ which gives:

$$
\begin{aligned}
& p_{-1,1}+p_{0,1}+p_{1,1} \neq p_{-1,-1}+p_{0,-1}+p_{1,-1} \text { and } \\
& p_{1,-1}+p_{1,0}+p_{1,1} \neq p_{-1,-1}+p_{-1,0}+p_{-1,1} .
\end{aligned}
$$




\section{Chapter 3}

\section{Kernel Equation \& Branches}

We saw in the last chapter that the only functional equation we have connecting all three generating functions is the fundamental form:

$$
-h(x, y) \pi(x, y)=h_{1}(x, y) \pi_{1}(x)+h_{2}(x, y) \pi_{2}(y)+h_{0}(x, y) \pi_{0,0} .
$$

Clearly, this equation has three unknown functions and to gather any meaningful information, we need to reduce the equation down so that it contains only two unknown functions, specifically $\pi_{1}(x)$ and $\pi_{2}(y)$. No doubt, the only way to achieve this task is to set the left hand side of the fundamental form equal to zero. This is why the function $h(x, y)$ is called the kernel function and $h(x, y)=0$ is called the kernel equation. And now we are in the business of studying the kernel equation $h(x, y)=0$ without using too advanced mathematical topics. Instead, we will use single variable complex analysis. 


\subsection{Kernel Equation \& Branch Points}

Let us define

$$
\mathcal{B}=\left\{(x, y) \in \mathbb{C}^{2}: h(x, y)=0\right\}
$$

to be the algebraic curve defined by the kernel equation of the random walk.

Now, $h(x, y)$ is a polynomial of degree at most two in variables $x$ and $y$. When $h(x, y)$ is reducible or of degree 1 in at least one of the variables $x$ and $y$, then the random walk is called singular [2]. Otherwise, $h(x, y)$ is irreducible and the random walk is called non-singular [2]. When a random walk is singular then the analysis involved is considered less demanding than the non-singular case. For this reason, all of our analysis will be under the assumption that the random walk is non-singular.

Note: When $h(x, y)$ is of degree one with respect to $y$, say, then $a(x)=0$ which is equivalent to $p_{i, 1}=0$, for every $i$. Similar results hold when $h(x, y)$ is of degree one with respect to $x$.

Let us first consider $h(x, y)$ as a polynomial in $y$, so that

$$
h(x, y)=a(x) y^{2}+b(x) y+c(x) .
$$

Then, for each fixed $x$ and $a(x) \neq 0, h(x, y)$ is a quadratic in the variable $y$ and the kernel equation gives us two solutions:

$$
Y_{ \pm}(x)=\frac{-b(x) \pm \sqrt{\delta(x)}}{2 a(x)} .
$$


where

$$
\delta(x)=b^{2}(x)-4 a(x) c(x) \text { is the discriminant. }
$$

Similarly, for each fixed $y$ and $\tilde{a}(y) \neq 0, h(x, y)$ is a quadratic in the variable $x$ and the kernel equation gives us two solutions:

$$
X_{ \pm}(y)=\frac{-\tilde{b}(y) \pm \sqrt{\tilde{\delta}(y)}}{2 \tilde{a}(y)}
$$

where

$$
\tilde{\delta}(y)=\tilde{b}^{2}(y)-4 a(y) c(y) \text { is the discriminant. }
$$

We immediately see that $Y_{ \pm}(x)$ and $X_{ \pm}(y)$ are two-valued functions with two branches each. Regrettably, analytic continuation and multivalued functions do not make a great couple. Problems can arise quickly since the value of a function prior to its journey along a path may not be the same once the function has returned back to its starting point [12]. Another issue with the two-valued functions is that we will be using them through out our analysis and we need to make sure their branches are well defined and we need to be able to specify which well defined branch we ought be using, depending on which area in the complex plane we are in. The classic approach to dealing with multivalued functions is to find their branch points and create branch cuts. It is on these branch cuts that the multivalued functions fail to be continuous, but all of their branches are specified and each one is well defined.

In what follows, since entirely symmetrical results hold for $X_{ \pm}(y)$ as they do 
for $Y_{ \pm}(x)$, we will only study $Y_{ \pm}(x)$ in detail. Now, the branch points of $Y_{ \pm}(x)$ are the values $x$ such that $\delta(x)=0$. Therefore, a good place to begin is to look at the equation $\delta(x)=0$ and try to deduce properties about its roots. In fact, the following theorem provides us with a host of information regarding the roots.

Theorem 3.1 [7]: If the polynomial $\delta(x)$ has degree four, and $M_{y} \neq 0$, then $\delta(x)$ has four real roots, two of which are inside the unit disk $\mathcal{D}_{x}(0,1)$, and two of which are outside the unit circle $\mathcal{D}_{x}(0,1)$.

Proof: By the Fundamental Theorem of Algebra, $\delta(x)$ has four roots. We need to show that these four roots lie on the real line. Since $\sum_{i, j} p_{i, j}=1$,

$$
p_{-1,1}+p_{0,1}+p_{1,1}+p_{-1,0}-\left(1-p_{0,0}\right)+p_{1,0}+p_{-1,-1}+p_{0,-1}+p_{1,-1}=0,
$$

and we have,

$$
\begin{aligned}
\delta(1)= & (b(1))^{2}-4 a(1) c(1) \\
= & \left(p_{-1,0}-\left(1-p_{0,0}\right)+p_{1,0}\right)^{2} \\
& -4\left(p_{-1,1}+p_{0,1}+p_{1,1}\right)\left(p_{-1,-1}+p_{0,-1}+p_{1,-1}\right) \\
= & \left(p_{-1,1}+p_{0,1}+p_{1,1}+p_{-1,-1}+p_{0,-1}+p_{1,-1}\right)^{2} \\
& -4\left(p_{-1,1}+p_{0,1}+p_{1,1}\right)\left(p_{-1,-1}+p_{0,-1}+p_{1,-1}\right) \\
= & \left(p_{-1,1}+p_{0,1}+p_{1,1}-\left(p_{-1,-1}+p_{0,-1}+p_{1,-1}\right)\right)^{2}>0 .
\end{aligned}
$$

Recall that $M_{y} \neq 0$ is equivalent to $p_{-1,1}+p_{0,1}+p_{1,1} \neq p_{-1,-1}+p_{0,-1}+p_{1,-1}$ and 
we notice that $\delta(1)=0$ precisely when $p_{-1,1}+p_{0,1}+p_{1,1}=p_{-1,-1}+p_{0,-1}+p_{1,-1}$.

Now let us consider the quadratic polynomial $b(x)=p_{-1,0}-\left(1-p_{0,0}\right) x+p_{1,0} x^{2}$, which has two roots given by:

$$
x_{1}^{b}, x_{2}^{b}=\frac{\left(1-p_{0,0}\right) \pm \sqrt{\left(1-p_{0,0}\right)^{2}-4 p_{-1,0} p_{1,0}}}{2 p_{-1,0}} .
$$

Seeing that, $\left(1-p_{0,0}\right)^{2}>\left(p_{-1,0}+p_{1,0}\right)^{2}$, since otherwise we would have $p_{-1,1}+$ $p_{0,1}+p_{1,1}=p_{-1,-1}+p_{0,-1}+p_{1,-1}=0$, we can deduce, by simple manipulation, that $\left(1-p_{0,0}\right)^{2}-4 p_{-1,0} p_{1,0}>0$.

Therefore, the discriminant of $b(x)$ is greater than zero, which implies that the two roots $x_{1}^{b}$ and $x_{2}^{b}$ are real and unique. Moreover, since $b(0)=p_{-1,0}>0$, $b(1)=\left(p_{-1,0}-\left(1-p_{0,0}\right)+p_{1,0}\right)<0$, and $b(\infty)=\infty$, have $0<x_{1}^{b}<1<x_{2}^{b}$. Note that $b(0)=0$ only when $p_{-1,0}=0$, in which case $x_{1}^{b}=0$. Also, $b(1)=0$ precisely when $p_{-1,1}+p_{0,1}+p_{1,1}=p_{-1,-1}+p_{0,-1}+p_{1,-1}$.

Furthermore, as $\delta(1)>0, \delta\left(x_{1}^{b}\right)=-4 a\left(x_{1}^{b}\right) c\left(x_{1}\right)^{b}<0$ and $\delta\left(x_{2}^{b}\right)=-4 a\left(x_{2}^{b}\right) c\left(x_{2}\right)^{b}>$ 0 , it is now clear, by employing basic properties of polynomials, that there exists roots $x_{2}$ and $x_{3}$, belonging to $\delta(x)$, such that, $x_{1}^{b}<x_{2}<1<x_{3}<x_{2}^{b}$. Now all we have left to do is to locate the last two roots of $\delta(x), x_{1}$ and $x_{4}$.

Let us denote $\delta_{0}=p_{-1,0}^{2}-4 p_{-1,1} p_{-1,-1}$ and $\delta_{4}=p_{1,0}^{2}-4 p_{1,1} p_{-1,1}$ to be the constant term and the coefficient of $x_{4}$, in $\delta(x)$, respectively.

Now,

1. If $\delta_{0}>0$ and $\delta_{4}>0$, we have $\delta(\infty)=\infty$ and, together with the above 
analysis, we get $0<x_{1}<x_{1}^{b}$ and $x_{2}^{b}<x_{4}<\infty$.

2. If $\delta_{0}<0$ and $\delta_{4}<0$, we have to consider

$\delta(-1)=\left(p_{-1,0}+\left(1-p_{0,0}\right)+p_{1,0}\right)^{2}-4\left(p_{-1,1}-p_{0,1}+p_{1,1}\right)\left(p_{-1,-1}-p_{0,-1}+p_{1,-1}\right)$

If the product $\left(p_{-1,1}-p_{0,1}+p_{1,1}\right)\left(p_{-1,-1}-p_{0,-1}+p_{1,-1}\right)>0$ then $\delta(-1)>$ $\left(p_{-1,0}+\left(1-p_{0,0}\right)+p_{1,0}\right)^{2}>0$.

When both $\left(p_{-1,1}-p_{0,1}+p_{1,1}\right)<0$ and $\left(p_{-1,-1}-p_{0,-1}+p_{1,-1}\right)<0$, then we get $\left|\left(p_{-1,1}-p_{0,1}+p_{1,1}\right)\right|<p_{0,1}$ and $\left|\left(p_{-1,-1}-p_{0,-1}+p_{1,-1}\right)\right|<p_{0,-1}$, which gives $\delta(-1)>\left(p_{-1,0}+\left(1-p_{0,0}\right)+p_{1,0}\right)^{2}>-4 p_{0,1} p_{0,-1}>0$.

Similarly, when both $\left(p_{-1,1}-p_{0,1}+p_{1,1}\right)>0$ and $\left(p_{-1,-1}-p_{0,-1}+p_{1,-1}\right)>$ 0 , then we get $\delta(-1)=\left(p_{-1,0}+\left(1-p_{0,0}\right)+p_{1,0}\right)^{2}-4\left(p_{-1,1}+p_{1,1}\right)\left(p_{-1,-1}+p_{1,-1}\right)>$ 0 .

It follows that since $\delta(-1)>0$ and $\delta(-\infty)<0,-\infty<x_{4}<-1<x_{1}<0$.

3. If $\delta_{0}>0$ and $\delta_{4}<0$ then, following the above results, and the fact that $\delta(\infty)=\infty$, we get $0<x_{1}<x_{1}^{b}$ and $-\infty x_{4}<-1$.

4. If $\delta_{0}<0$ and $\delta_{4}>0$ then $-1<x_{1}<0$ and $x_{2}^{b}<x_{4}<\infty$.

5. If $\delta_{0}=0$ then we have $x_{1}=0$.

6. If $\delta_{4}=0$ then $\delta(x)$ reduces to a cubic polynomial and, when $\delta_{0}>0$, we get the three real roots $0<x_{1}<x_{1}^{b}<x_{2}<1<x_{3}<\infty$. 
Before we conclude our proof, there should be a note concerning the case when $\delta_{4}=0$, or, equivalently, when the polynomial $\delta(x)$ has degree three. Our efforts to classify the roots of $\delta(x)$ was motivated by our goal to find the branch points of $Y_{ \pm}(x)$. Since, in this case, $Y_{ \pm}(x)$ has an odd number of branch points, and we need an even number of branch points to create our branch cuts, we define the fourth branch point to be $x_{4}=\infty$. This can be more rigorously demonstrated by using the inversion $x=\frac{1}{\zeta}$ and showing that $\zeta=0$ is a branch point of $Y_{ \pm}\left(\frac{1}{\zeta}\right)$. Symmetrically identical results hold for $\tilde{\delta}(y)$ and the branch points $y_{1}, y_{2}, y_{3}$ and $y_{4}$.

The previous proof leads to the following corollary, which enables us to further localize the roots, $x_{1}, x_{2}, x_{3}$, and $x_{4}$, of $\delta(x)$, when $\delta(x)$ has degree four:

Corollary 3.2 [7]: If $p_{1,0}^{2}>4 p_{1,1} p_{1,-1}$ and $p_{-1,0}^{2} \geq 4 p_{-1,1} p_{-1,-1}$, then $0 \leq x_{1}<$ $x_{2}<1<x_{3}<x_{4}<\infty$ and $\delta(x)<0$ if and only if $x \in\left(x_{1}, x_{2}\right) \cup\left(x_{3}, x_{4}\right)$.

Proof: If $p_{1,0}^{2}>4 p_{1,1} p_{1,-1}$, then this coincides with $\delta_{4}>0$, where $\delta_{4}$ is defined in the proof of Theorem 3.1, and, as previously shown, we have $1<x_{3}<x_{4}<\infty$. If $p_{-1,0}^{2}>4 p_{-1,1} p_{-1,-1}$, then this coincides with $\delta_{0}(x)>0$, and we get, as also previously shown, $0<x_{1}<x_{2}<1$. If $p_{-1,0}^{2}=4 p_{-1,1} p_{-1,-1}$, then $\delta(x)$ is reducible, since $\delta_{0}=0$, and we can factor out an $x$, which then gives $0=x_{1}<x_{2}<1$.

It is clear, based on previous results and the geometric properties of a quartic function, that $\delta(x)<0$ if and only if $x \in\left(x_{1}, x_{2}\right) \cup\left(x_{3}, x_{4}\right)$. Symmetrically 
identical results hold for $y_{1}, y_{2}, y_{3}$ and $y_{4}$.

Remark: It should be noted that when $M_{y}=0$ and $M_{x}<0$, then $0<x_{1}<$ $x_{2}=1<x_{3}<x_{4}<\infty$ and when $M_{y}=0$ and $M_{x}>0$, then $0<x_{1}<x_{2}<x_{3}=$ $1<x_{4}<\infty$. In the heavy-tail case, when both $M_{y}=0$ and $M_{x}=0$, then we get a double root for the discriminant: $0<x_{1}<x_{2}=x_{3}=1<x_{4}<\infty$. These assertions can be easily proven [17].

We know that $Y_{ \pm}(x)$ is a multivalued function, and the roots of the discriminant $x_{1}, x_{2}, x_{3}$, and $x_{4}$, are branch points. Accordingly, we make our branch cuts, in the $\mathbb{C}_{x}$ plane, to be the two intervals $\left[x_{1}, x_{2}\right]$ and $\left[x_{3}, x_{4}\right]$, and, as long as we do not cross these branch cuts, we will remain on one of the two branches of $Y_{ \pm}(x)$. However, the story does not end here. 


\subsection{The Branches of $Y_{ \pm}(x) \& X_{ \pm}(y)$}

Taking a step back, let us briefly look at the square root function $\sqrt{z}$ in the complex plane. The typical approach is to show that $z=0$ and $z=\infty$ are branch points, and then make a cut along the entire negative real line, in the complex plane. Thus, for $z \in \mathbb{C} \backslash\{z: \Re(z) \leq 0, \Im(z)=0\}, \sqrt{z}$ is analytic, and we can choose the branch which defines $\sqrt{z}$ so that $\sqrt{1}=1$. Furthermore, the branch $\sqrt{z}$ fails to be continuous, and hence analytic, when $z \in\{z: \Re(z) \leq 0, \Im(z)=0\}$, $\sqrt{z}$. What happens when we try to define $\sqrt{g(z)}$ for $z \in \mathbb{C}$ ?

Example: Consider the function $f(z)=\sqrt{z(z-1)}$ for $z \in \mathbb{C}$. It is obvious that $z=0$ and $z=1$ are branch point of the function and our branch cut will be the real interval $[0,1]$. Clearly, $\sqrt{z(z-1)}$ fails to be analytic on this interval. However, $\sqrt{z(z-1)}$ will also fail to be analytic if $\Re(z(z-1)) \leq 0$ and $\Im(z(z-1))=0$. If we let $z=x+i y$, then $(z(z-1))=x^{2}-y^{2}-x+i(2 x y-y)$ and we immediately see that the function also fails to be analytic for all $x$ and all $y$ such that $x^{2}-y^{2}-x \leq 0$ and for $x=\frac{1}{2}, y=0$. Notice that $x=\frac{1}{2}$ and $y=0$ are included in the branch cut.

The above example demonstrates that when defining the region of analyticity for both $Y_{-}(x)$ and $Y_{+}(x)$, finding the branch cuts is not enough. We need to determine the curves where $\Re(\delta(x)) \leq 0$ and $\Im(\delta(x))=0$. Let us focus on the 
function $\delta(x)$ momentarily.

Assume $p_{-1,0}^{2} \geq 4 p_{-1,1} p_{-1,-1}$ and $p_{1,0}^{2} \geq 4 p_{1,1} p_{-1,1}$.

Let $\delta=\delta_{4} x^{4}+\delta_{3} x^{3}+\delta_{2} x^{2}+\delta_{1} x^{1}+\delta_{0}$, where,

$$
\begin{aligned}
& \delta_{4}=p_{1,0}^{2}-4 p_{1,1} p_{-1,1} \geq 0 \\
& \delta_{3}=2 p_{1,0}\left(p_{0,0}-1\right)-4\left(p_{1,1} p_{0,-1}+P_{0,1} p_{1,-1}\right)<0 \\
& \delta_{2}=\left(p_{0,0}-1\right)^{2}+2 p_{1,0} p_{-1,0}-4\left(p_{1,1} p_{-1,-1}+p_{1,-1} p_{-1,1}+p_{0,1} p_{0,-1}\right) \\
& \delta_{1}=2 p_{-1,0}\left(p_{0,0}-1\right)-4\left(p_{-1,1} p_{0,-1}+P_{0,1} p_{-1,-1}\right)<0 \\
& \delta_{0}=p_{-1,0}^{2}-4 p_{-1,1} p_{-1,-1} \geq 0 .
\end{aligned}
$$

Now take an $x \in \mathbb{C}_{x}$. Then $x$ can be written as $x=u+i v$, with $u, v \in \mathbb{R}$, and $\delta(x)=A(u, v)+i B(u, v)$, where

$$
\begin{aligned}
& A(u, v)=\delta_{4}\left(u^{4}-6 u^{2} v^{2}+v^{4}\right)+\delta_{3} u\left(u^{2}-3 v^{2}\right)+\delta_{2}\left(u^{2}-v^{2}\right)+\delta_{1} u+\delta_{0}, \\
& B(u, v)=v\left[\delta^{\prime}(u)-\left(4 \delta_{4} u+\delta_{3}\right) v^{2}\right] .
\end{aligned}
$$

The derivative $\delta^{\prime}(x)$ is a cubic polynomial with three real roots, $\hat{x}_{1}, \hat{x}_{2}$, and $\hat{x}_{3}$, such that, $0 \leq x_{1}<\hat{x}_{1}<x_{2}<\hat{x}_{2}<x_{3}<\hat{x}_{3}<x_{4}$. Furthermore, $\delta^{\prime \prime \prime}(x)=0$, gives us $x=\frac{-\delta_{3}}{4 \delta_{4}}>0$ which is the inflection point of $\delta^{\prime}(x)$ and therefore, $\hat{x}_{1}<\frac{-\delta_{3}}{4 \delta_{4}}<\hat{x}_{3}$.

Determining the value for which $\Im(\delta(x))=0$ is equivalent to solving $B(u, v)=$ 0 . The first obvious solution is $v=0$, in with case, $A(u, 0)=\delta(u) \leq 0$ if and only 
if $u \in\left[x_{1}, x_{2}\right] \cup\left[x_{3}, x_{4}\right]$.

If $v \neq 0$, then the other solution to $B(u, v)=0$ is $v= \pm \sqrt{\frac{\delta^{\prime}(u)}{4 \delta_{4}+\delta_{3}}}$,

for $u<\hat{x}_{1}, \quad u>\hat{x}_{3} \quad$ and $\left\{\begin{array}{lll}\hat{x}_{2}<u<\frac{-\delta_{3}}{4 \delta_{4}} & \text { if } \quad \hat{x}_{2}<\frac{-\delta_{3}}{4 \delta_{4}} \\ \frac{-\delta_{3}}{4 \delta_{4}}<u<\hat{x}_{2} & \text { if } \quad \hat{x}_{2}>\frac{-\delta_{3}}{4 \delta_{4}}\end{array}\right.$.

Notice that $A(u, v)=0$ and $B(u, v)=0$ exactly when $v=0$ and $u$ is any one of the four roots of $\delta(x)$. This tells us that the sign of $A(u, v)$ stays constant over any one of the half branches of $B(u, v)=0$, in both the upper half plane and the lower half plane. Now we consider the following three curves [7]:

$$
\begin{aligned}
& \mathfrak{C}_{1}=\left\{u+i v: u \leq \hat{x}_{1} \text { and } v= \pm \sqrt{\frac{\delta^{\prime}(u)}{4 \delta_{4}+\delta_{3}}}\right\}, \\
& \mathfrak{C}_{3}=\left\{u+i v: u \geq \hat{x}_{3} \text { and } v= \pm \sqrt{\frac{\delta^{\prime}(u)}{4 \delta_{4}+\delta_{3}}}\right\}, \\
& \mathfrak{C}_{2}=\left\{u+i v: \hat{x}_{2}<u<\frac{-\delta_{3}}{4 \delta_{4}} \text { and } v= \pm \sqrt{\frac{\delta^{\prime}(u)}{4 \delta_{4}+\delta_{3}}}\right\}, \text { if } \hat{x}_{2}<\frac{-\delta_{3}}{4 \delta_{4}}, \text { or, } \\
& \mathfrak{C}_{2}=\left\{u+i v: \frac{-\delta_{3}}{4 \delta_{4}}<u<\hat{x}_{2} \text { and } v= \pm \sqrt{\frac{\delta^{\prime}(u)}{4 \delta_{4}+\delta_{3}}}\right\}, \text { if } \hat{x}_{2}>\frac{-\delta_{3}}{4 \delta_{4}} .
\end{aligned}
$$

Note that for large $u, v^{2}=\frac{\delta^{\prime}(u)}{4 \delta_{4}+\delta_{3}} \sim u^{2}$ and this implies that, for large $u, A(u, v)$ $\sim \delta_{4}\left(u^{4}-6 u^{2} v^{2}+v^{4}\right) \sim-4 \delta_{4} u^{4}<0$. Therefore, along the curves $\mathfrak{C}_{1}$ and $\mathfrak{C}_{3}$, $A(u, v) \leq 0$.

For the curve $\mathfrak{C}_{2}, u$ is bounded, so that for large $v, A(u, v) \sim \delta_{4} v^{4}$. Since $\delta_{4}$ is always positive, $A(u, v) \geq 0$.

In order to continue $\sqrt{\delta(x)}$ as an analytic function in the maximal domain 
in the plane, we have to consider its other branch, $-\sqrt{\delta(x)}$. We now have all the necessary ingredients required to analytically continue $\sqrt{\delta(x)}$ in the cut plane $\mathbb{C}_{x} \backslash\left[x_{1}, x_{2}\right] \cup\left[x_{3}, x_{4}\right]$. Let $z^{+}=\Re(z)+i|\Im(z)|$, and define $\xi$ by [7]:

$\xi(x)= \begin{cases}+\sqrt{\delta(x)}, & \text { if } x \in\left\{z: \Re(z) \leq \hat{x}_{1}, \Im\left(\delta\left(z^{+}\right)\right)<0\right\} \cup\left(-\infty, x_{1}\right) \\ +\sqrt{\delta(x)}, & \text { if } x \in\left\{z: \Re(z) \geq \hat{x}_{3}, \Im\left(\delta\left(z^{+}\right)\right)>0\right\} \cup\left(x_{4}, \infty\right) . \\ -\sqrt{\delta(x)} & \text { otherwise }\end{cases}$

Therefore, $\xi(x)$ is the analytic continuation of $\sqrt{\delta(x)}$, to the cut plane, $\mathbb{C}_{x} \backslash\left[x_{1}, x_{2}\right] \cup$ $\left[x_{3}, x_{4}\right]$, where passing from one branch, $\sqrt{\delta(x)}$, to the other branch, $-\sqrt{\delta(x)}$, when $\delta(x) \in(-\infty, 0]$, makes $\xi(x)$ analytic in the specified domain.

The next step is to classify the singularities of the functions $Y_{ \pm}(x)$. When we look at the denominator of $Y_{ \pm}(x)$, we immediately see that the two roots of the quadratic polynomial $a(x)$, denoted by $\alpha$ and $\beta$, are the singularities of $Y_{ \pm}(x)$. These two roots are real, as the discriminant of $a(x)$, namely, $p_{1,0}^{2}-4 p_{1,1} p_{-1,1} \geq 0$, by assumption. Furthermore, the two roots are neither essential singularities nor branch points of $Y_{ \pm}(x)$. Therefore, the two roots are either removable singularities or poles of $Y_{ \pm}(x)$.

By rationalizing the denominator of $Y_{+}(x)$, and noting that $a(x), b(x)$ and $c(x)$ are continuous in $\mathbb{C}_{x}$, we have, for $x \in \mathbb{C}_{x} \backslash\left[x_{1}, x_{2}\right] \cup\left[x_{3}, x_{4}\right]$ :

$\lim _{x \rightarrow \alpha} Y_{+}(x)=\lim _{x \rightarrow \alpha} \frac{2 c(x)}{-b(x)-\sqrt{b^{2}(x)-4 a(x) c(x)}}=\frac{c(\alpha)}{b(\alpha)}<\infty$. 
Since $\gamma \in \mathbb{C}$ is a removable singularity of $f(z)$ if and only if for $z \in \mathbb{C}, \lim _{z \rightarrow \gamma} f(z)$ exists [12], $\alpha$ is a removable singularity of $Y_{+}(x)$. The same result can be drawn for $\beta$. Furthermore, $Y_{+}(x)$ has two roots which are the solutions to $c(x)=0$. Notice that $b(x) \neq 0$ since we are taking the limits in the cut plane, and we have previously shown that the roots of $b(x)$ lie in $\left[x_{1}, x_{2}\right] \cup\left[x_{3}, x_{4}\right]$. In conclusion, $Y_{+}(x)$ has no poles and at most two zeros.

On the other hand, by rationalizing the denominator of $Y_{-}(x)$ and with a few simple manipulations, we get $Y_{-}(x)=\frac{c(x)}{a(x) Y_{+}(x)}$. It is clear that $Y_{-}(x)$ has at most two simple poles, which are the solutions to $a(x)=0$, and no zeros.

We can now state the following two theorems:

Theorem 3.3: [7] If $\delta(x)$ has degree four, then the function $Y^{*}(x)$ defined by $Y^{*}(x)= \begin{cases}Y_{+}(x), & \text { if } x \in\left\{z: \Re(z) \leq x_{2}, \Im\left(\delta\left(z^{+}\right)\right)<0\right\} \cup\left(-\infty, x_{1}\right) \\ Y_{+}(x), & \text { if } x \in\left\{z: \Re(z) \geq x_{3}, \Im\left(\delta\left(z^{+}\right)\right)>0\right\} \cup\left(x_{4}, \infty\right) \\ Y_{-}(x) & \text { otherwise }\end{cases}$ is analytic in $\mathbb{C}_{x} \backslash\left[x_{1}, x_{2}\right] \cup\left[x_{3}, x_{4}\right]$.

Theorem 3.4: [7] If $\delta(x)$ has degree four, then the function defined by $Y_{*}(x)$

$$
Y_{*}(x)= \begin{cases}Y_{-}(x), & \text { if } x \in\left\{z: \Re(z) \leq x_{2}, \Im\left(\delta\left(z^{+}\right)\right)<0\right\} \cup\left(-\infty, x_{1}\right) \\ Y_{-}(x), & \text { if } x \in\left\{z: \Re(z) \geq x_{3}, \Im\left(\delta\left(z^{+}\right)\right)>0\right\} \cup\left(x_{4}, \infty\right) \\ Y_{+}(x) & \text { otherwise }\end{cases}
$$

is meromorphic in $\mathbb{C}_{x} \backslash\left[x_{1}, x_{2}\right] \cup\left[x_{3}, x_{4}\right]$ with potential singularities at the solutions 
to $a(x)$.

Symmetrical results of Theorem 3.3 and Theorem 3.4 hold for both $X^{*}(y)$ and $X_{*}(y)$.

Furthermore, we can express both $Y^{*}(x)$ and $Y_{*}(x)$ as:

$Y^{*}(x)=\frac{-b(x)+\sqrt{\xi(x)}}{2 a(x)}$ and

$Y_{*}(x)=\frac{-b(x)-\sqrt{\xi(x)}}{2 a(x)}$.

Some literature regarding random walks uses the definition and notation of $Y^{*}(x)$ and $Y_{*}(x)$ as defined above. However, other written works, including classical literature, defines the branches of $Y_{ \pm}(x)$ based on the size of their moduli. Certain results are easier to extract when defining the branches in the aforementioned fashion of modulus size. Here we present an alternate definition of the branches $Y^{*}(x)$ and $Y_{*}(x)$.

In what follows, we use source [17] with some modifications. Again we consider the kernel equation $h(x, y)=a(x) y^{2}+b(x) y+c(x)=0$, and assume that $h(x, y)$ is a polynomial of degree two in both the variables $x$ and $y$. Then, for each fixed $x$ and $a(x) \neq 0, h(x, y)=0$ gives two solutions for $y$ :

$$
Y_{ \pm}(x)=\frac{-b(x) \pm \sqrt{b(x)^{2}-4 a(x) c(x)}}{2 a(x)} .
$$

The goal here is to extract more information from the two branches $Y_{ \pm}(x)$ by employing an alternative method in expressing the already introduced functions 
$Y^{*}(x)$ and $Y_{*}(x)$.

Through basic manipulation and completing the square, we have, for each fixed $x, a(x) \neq 0$, and $a(x) y^{2}+b(x) y+c(x)=0:$

$$
\left(y(x)+\frac{b(x)}{a(x)}\right)^{2}=\left(\frac{b(x)}{2 a(x)}\right)^{2}-\frac{c(x)}{a(x)} .
$$

To simplify the written notations for the functions used in this section, we define $y(x) \equiv y, u(x) \equiv u, v(x) \equiv v, u_{1}(x) \equiv u_{1}, u_{2}(x) \equiv u_{2}, v_{1}(x) \equiv v_{1}, v_{2}(x) \equiv v_{2}$, $F(x) \equiv F$, and $G(x) \equiv G$, and then we let:

$$
\begin{gathered}
y=u+i v, \quad \frac{b(x)}{2 a(x)}=u_{1}+i v_{1}, \quad-\frac{c(x)}{a(x)}=u_{2}+i v_{2}, \quad \text { and } \\
\left(\frac{b(x)}{2 a(x)}\right)^{2}-\frac{c(x)}{a(x)}=G+i F .
\end{gathered}
$$

Now, (3.2) gives us $\left(u_{1}+i v_{1}\right)^{2}+\left(u_{2}+i v_{2}\right)=u_{1}^{2}-v_{1}^{2}+u_{2}+i\left(2 u_{1} v_{1}+v_{2}\right)$, and therefore,

$$
\begin{aligned}
& G=u_{1}^{2}-v_{1}^{2}+u_{2} \\
& F=\left(2 u_{1} v_{1}+v_{2}\right) .
\end{aligned}
$$

However, the left hand side of $(3.1)$ gives $\left(u+i v+2 u_{1}+i 2 v_{1}\right)^{2}$ and by comparing 
the real and imaginary parts, we get:

$$
\begin{aligned}
& G=\left(u+u_{1}\right)^{2}-\left(v+v_{1}\right)^{2}, \\
& F=2\left(u+u_{1}\right)\left(v+v_{1}\right) .
\end{aligned}
$$

If we assume that $F \neq 0$ then:

$$
v+v_{1}=\frac{F}{2\left(u+u_{1}\right)},
$$

and,

$$
\begin{aligned}
2\left(u+u_{1}\right)^{2} & =\left(u+u_{1}\right)^{2}-\left(v+v_{1}\right)^{2}+\left(u+u_{1}\right)^{2}+\left(v+v_{1}\right)^{2} \\
& =\left(u+u_{1}\right)^{2}-\left(v+v_{1}\right)^{2}+\sqrt{\left(\left(u+u_{1}\right)^{2}+\left(v+v_{1}\right)^{2}\right)^{2}} \\
& =G+\sqrt{\left(\left(u+u_{1}\right)^{2}-\left(v+v_{1}\right)^{2}\right)^{2}+4\left(u+u_{1}\right)^{2}\left(v+v_{1}\right)^{2}} \\
& =G+\sqrt{G^{2}+F^{2}},
\end{aligned}
$$

which gives,

$$
\left(u+u_{1}\right)^{2}=\frac{G+\sqrt{G^{2}+F^{2}}}{2} \equiv D^{+} .
$$


We also define $\frac{-G+\sqrt{G^{2}+F^{2}}}{2} \equiv D^{-}$.

Recalling that $\sqrt{x^{2}}=|x|$, we have,

$u+u_{1}= \pm \sqrt{D^{+}}$, and,

$v+v_{1}=\frac{F}{2 \sqrt{D^{+}}}=\frac{F \sqrt{D^{-}}}{2 \sqrt{D^{+}} \sqrt{D^{-}}}=\frac{F}{|F|} \sqrt{D^{-}}$.

Now we let

$$
\begin{aligned}
& u_{ \pm}=-u_{1} \pm \sqrt{D^{+}}, \\
& v_{ \pm}=-v_{1} \pm \frac{F}{|F|} \sqrt{D^{-}} .
\end{aligned}
$$

Replacing the functions $u$ and $v$, in $y=u+i v$, with the functions $u_{ \pm}$and $v_{ \pm}$, renders the two solutions of the equation $a(x) y(x)^{2}+b(x) y(x)+c(x)=0$, given by:

$$
\begin{aligned}
& Y_{-}(x)=\left(-u_{1}-\sqrt{D^{+}}\right)+i\left(-v_{1}-\frac{F}{|F|} \sqrt{D^{-}}\right) \\
& Y_{+}(x)=\left(-u_{1}+\sqrt{D^{+}}\right)+i\left(-v_{1}+\frac{F}{|F|} \sqrt{D^{-}}\right) .
\end{aligned}
$$

Depending on the sign and value of the functions $F$ and $G$, we have the following cases:

1. If $F>0$ then $|F|=F$ so that $\Im\left(Y_{-}(x)\right)=-v_{1}-\sqrt{D^{-}}$and $\Im\left(Y_{+}(x)\right)=$ $-v_{1}+\sqrt{D^{-}}$. 
2. If $F<0$ then $|F|=-F$ so that $\Im\left(Y_{-}(x)\right)=-v_{1}+\sqrt{D^{-}}$and $\Im\left(Y_{+}(x)\right)=$ $-v_{1}-\sqrt{D^{-}}$.

3. If $F=0$ then either $v=v_{1}$ or $u=u_{1}$. If $v=v_{1}$ then $G=\left(u+u_{1}\right)^{2}>\geq$ and $u=-u_{1} \pm \sqrt{G}$. If $u=u_{1}$ then $G=-\left(v+v_{1}\right)^{2}<0$ and $v=v_{1} \pm \sqrt{-G}$

To summarize, we can define $Y_{-}(x)$ and $Y_{+}(x)$ as follows [17]:

$$
\begin{aligned}
& Y_{-}(x)= \begin{cases}\left(u_{1}-\sqrt{D^{+}}\right)+i\left(-v_{1}-\sqrt{D^{-}}\right), & \text {if } F>0 \\
\left(u_{1}-\sqrt{D^{+}}\right)+i\left(-v_{1}+\sqrt{D^{-}}\right), & \text {if } F<0 \\
-u_{1}+i\left(-v_{1}-\sqrt{-G}\right), & \text { if } F=0 \text { and } G<0 \\
\left(-u_{1}-\sqrt{G}\right)-i v_{1}, & \text { if } F=0 \text { and } G \geq 0\end{cases} \\
& Y_{+}(x)= \begin{cases}\left(u_{1}+\sqrt{D^{+}}\right)+i\left(-v_{1}+\sqrt{D^{-}}\right), & \text {if } F>0 \\
\left(u_{1}+\sqrt{D^{+}}\right)+i\left(-v_{1}-\sqrt{D^{-}}\right), & \text {if } F<0 \\
-u_{1}+i\left(-v_{1}+\sqrt{-G}\right), & \text { if } F=0 \text { and } G<0 \\
\left(-u_{1}+\sqrt{G}\right)-i v_{1}, & \text { if } F=0 \text { and } G \geq 0\end{cases}
\end{aligned}
$$

Proposition 3.5 [17]: $\left|Y_{-}(x)\right|=\left|Y_{+}(x)\right|$ if and only if $x \in\left[x_{1}, x_{2}\right] \cup\left[x_{3}, x_{4}\right]$.

Proof: Let $x \in\left(x_{1}, x_{2}\right) \cup\left(x_{3}, x_{4}\right)$. Then $b(x)^{2}-4 a(x) c(x)<0$ and therefore $Y_{-}(x)$ and $Y_{+}(x)$ are complex conjugates, so that $\left|Y_{-}(x)\right|=\left|Y_{+}(x)\right|$. When $x \in\left\{x_{1}, x_{2}, x_{3}, x_{4}\right\}$, then $b(x)^{2}-4 a(x) c(x)=0$, so that $Y_{-}(x)=Y_{+}(x)$, which obviously implies that $\left|Y_{-}(x)\right|=\left|Y_{+}(x)\right|$. Conversely, if $\left|Y_{-}(x)\right|=\left|Y_{+}(x)\right|$, then either, based on the definition of $Y_{ \pm}(x), Y_{-}(x)$ and $Y_{+}(x)$ are complex conjugates, and hence, $x \in\left(x_{1}, x_{2}\right) \cup\left(x_{3}, x_{4}\right)$, or $Y_{-}(x)=Y_{+}(x)$, which implies that 
$x \in\left\{x_{1}, x_{2}, x_{3}, x_{4}\right\}$.

Remark: If $F=0$ and $G=0$, then $Y_{-}(x)=Y_{+}(x)=-\left(u_{1}+i v_{1}\right)$.

Proposition 3.6 [17]: Let $x \in \mathbb{C}_{x} \backslash\left[x_{1}, x_{2}\right] \cup\left[x_{3}, x_{4}\right]$ and define functions $\Psi_{ \pm}(x) \equiv$ $\Psi_{ \pm}$to be:

$\Psi_{+}=u_{1} \sqrt{D^{+}}+v_{1} \sqrt{D^{-}}, \Psi_{-}=u_{1} \sqrt{D^{+}}-v_{1} \sqrt{D^{-}}$.

Then,

1. $\left|Y_{-}(x)\right|<\left|Y_{+}(x)\right|$ if and only if $\Psi_{+}<0$ if $F>0$,

2. $\left|Y_{-}(x)\right|<\left|Y_{+}(x)\right|$ if and only if $\Psi_{-}<0$ if $F<0$,

3. $\left|Y_{-}(x)\right|<\left|Y_{+}(x)\right|$ if and only if $v_{1}<0$ if $F=0$ and $G<0$,

4. $\left|Y_{-}(x)\right|<\left|Y_{+}(x)\right|$ if and only if $u_{1}<0$ if $F=0$ and $G>0$.

Proof: Recall that $\left|Y_{ \pm}(x)\right|=\sqrt{\left(\Re\left(Y_{ \pm}(x)\right)^{2}+\left(\Im\left(Y_{ \pm}(x)\right)^{2}\right.\right.}$. Therefore, we have the following:

If $F>0$, then

$$
\begin{aligned}
& \left|Y_{-}(x)\right|=u_{1}^{2}+2 u_{1} \sqrt{D^{+}}+D^{+}+v_{1}^{2}+2 v_{1} \sqrt{D^{-}}+D^{-}, \\
& \left|Y_{+}(x)\right|=u_{1}^{2}-2 u_{1} \sqrt{D^{+}}+D^{+}+v_{1}^{2}-2 v_{1} \sqrt{D^{-}}+D^{-}
\end{aligned}
$$

and $\left|Y_{-}(x)\right|<\left|Y_{+}(x)\right|$ if and only if $u_{1} \sqrt{D^{+}}+v_{1} \sqrt{D^{-}}<-\left(u_{1} \sqrt{D^{+}}+v_{1} \sqrt{D^{-}}\right)$, if 
and only if, $u_{1} \sqrt{D^{+}}+v_{1} \sqrt{D^{-}}, 0$, i.e., if and only if, $\Psi_{+}<0$.

If $F<0$, then

$\left|Y_{-}(x)\right|=u_{1}^{2}+2 u_{1} \sqrt{D^{+}}+D^{+}+v_{1}^{2}-2 v_{1} \sqrt{D^{-}}+D^{-}$,

$\left|Y_{+}(x)\right|=u_{1}^{2}-2 u_{1} \sqrt{D^{+}}+D^{+}+v_{1}^{2}+2 v_{1} \sqrt{D^{-}}+D^{-}$

and $\left|Y_{-}(x)\right|<\left|Y_{+}(x)\right|$, if and only if, $u_{1} \sqrt{D^{+}}-v_{1} \sqrt{D^{-}}<-\left(u_{1} \sqrt{D^{+}}-v_{1} \sqrt{D^{-}}\right)$, if and only if, $u_{1} \sqrt{D^{+}}-v_{1} \sqrt{D^{-}}, 0$, i.e., if and only if, $\Psi_{-}<0$.

If $F=0$ and $G<0$, then

$\left|Y_{-}(x)\right|=u_{1}^{2}+v_{1}^{2}-2 v_{1} \sqrt{-G}-G$,

$\left|Y_{+}(x)\right|=u_{1}^{2}+v_{1}^{2}+2 v_{1} \sqrt{-G}-G$,

and $\left|Y_{-}(x)\right|<\left|Y_{+}(x)\right|$ if and only if $-v_{1}<v_{1}$, i.e., if and only if, $v_{1}<0$.

If $F=0$ and $G>0$, then

$\left|Y_{-}(x)\right|=u_{1}^{2}+v_{1}^{2}+2 u_{1} \sqrt{G}+G$,

$\left|Y_{+}(x)\right|=u_{1}^{2}+v_{1}^{2}-2 u_{1} \sqrt{G}+G$,

and $\left|Y_{-}(x)\right|<\left|Y_{+}(x)\right|$, if and only if, $-u_{1}<u_{1}$, i.e., if and only if, $u_{1}<0$.

We are now in a strong position to formally define the two branches of $Y_{ \pm}(x)$ for 
$x \in \mathbb{C}_{x} \backslash\left[x_{1}, x_{2}\right] \cup\left[x_{3}, x_{4}\right]$. We have, for $a(x) \neq 0$,

$$
\begin{gathered}
Y_{0}(x)= \begin{cases}Y_{-}(x), & \text { if } F \geq 0 \text { and } \Psi_{+}<0, \text { or, } F \leq 0 \text { and } \Psi_{-}<0 \\
Y_{+}(x), & \text { if } F \geq 0 \text { and } \Psi_{+}<0, \text { or, } F \leq 0 \text { and } \Psi_{-}<0\end{cases} \\
Y_{1}(x)= \begin{cases}Y_{+}(x), & \text { if } F \geq 0 \text { and } \Psi_{+}<0, \text { or, } F \leq 0 \text { and } \Psi_{-}<0 \\
Y_{-}(x), & \text { if } F \geq 0 \text { and } \Psi_{+}<0, \text { or, } F \leq 0 \text { and } \Psi_{-}<0\end{cases}
\end{gathered}
$$

The above expressions can be further simplified by noting that the choice of either $Y_{-}(x)$ or $Y_{+}(x)$ is purely based on their moduli [17]. Therefore,

$$
\begin{gathered}
Y_{0}(x)= \begin{cases}Y_{-}(x), & \text { if }\left|Y_{-}(x)\right| \leq\left|Y_{+}(x)\right| \\
Y_{+}(x), & \text { if }\left|Y_{-}(x)\right|>\left|Y_{+}(x)\right|\end{cases} \\
Y_{1}(x)= \begin{cases}Y_{+}(x), & \text { if }\left|Y_{-}(x)\right| \leq\left|Y_{+}(x)\right| \\
Y_{-}(x), & \text { if }\left|Y_{-}(x)\right|>\left|Y_{+}(x)\right|\end{cases}
\end{gathered}
$$

We notice that, despite intuition, the fact that $Y_{0}(x)$ is the function with the smaller modulus does not imply that $Y_{0}(x)=Y_{-}(x)$ [17]. Again, $\left|Y_{-}(x)\right| \leq\left|Y_{+}(x)\right|$ for $x \in \mathbb{C}_{x} \backslash\left[x_{1}, x_{2}\right] \cup\left[x_{3}, x_{4}\right]$, with equality taking place only for $x \in\left[x_{1}, x_{2}\right] \cup$ $\left[x_{3}, x_{4}\right]$. Furthermore, $Y_{0}(x)$ is an analytic function and $Y_{1}(x)$ is a meromorphic function, both in $\mathbb{C}_{x} \backslash\left[x_{1}, x_{2}\right] \cup\left[x_{3}, x_{4}\right]$. In fact, as already shown, $Y_{0}(x)$ has at most two roots and no poles in $\mathbb{C}_{x} \backslash\left[x_{1}, x_{2}\right] \cup\left[x_{3}, x_{4}\right]$ and $Y_{1}(x)$ has at most two poles and no roots in $\mathbb{C}_{x} \backslash\left[x_{1}, x_{2}\right] \cup\left[x_{3}, x_{4}\right]$.

The following is one of the most important theorems that we will take full advantage of since it offers us some definitive properties of $Y_{0}(x)$ and $Y_{1}(x)$ on the 
unit circle.

Theorem 3.7 [2]: For $|x|=1,\left|Y_{0}(x)\right| \leq 1$ and $\left|Y_{1}(x)\right| \geq 1$, with equality only possibly for $x= \pm 1$. Moreover, for $x=1$, we have

$$
\begin{aligned}
& Y_{0}(1)=\min \left(1, \frac{c(1)}{a(1)}\right) \\
& Y_{1}(x)=\max \left(1, \frac{c(1)}{a(1)}\right) .
\end{aligned}
$$

Proof: Omitted 


\subsection{The contours $\partial D_{y} \& \partial D_{x}$}

We now have an idea of how $Y_{0}(x)$ and $Y_{1}(x)$ behave on the unit circle. We know that $\left|Y_{0}(x)\right| \leq 1$ and $\left|Y_{0}(x)\right| \geq 1$. What happens on the cut $\left[x_{1}, x_{2}\right]$ ? The following is a geometric explanation of how we can crudely interpret the image of $Y_{ \pm}(x)$ as $x$ varies through the line segment $\left[x_{1}, x_{2}\right]$.

As noted, when $x \in\left(x_{1}, x_{2}\right), \delta(x)<0$ and it is clear that the two roots of $Y_{ \pm}(x)$ are complex conjugate. Therefore, the image of the segment $\left[x_{1}, x_{2}\right]$ is a closed contour, denoted by $\partial D_{y}$, that is symmetric to the horizontal axis in the $\mathbb{C}_{y}$ plane. Moreover, the closed contour $\partial D_{y}$ outlines a bounded domain $D_{y}$ in the $\mathbb{C}_{y}$ plane. In fact, we can parametrize the contour $\partial D_{y}$ by:

$$
\left\{\frac{p_{1,0} x^{2}-\left(1-p_{0,0}\right) x+p_{-1,0}}{2\left(p_{1,1}, x^{2}+p_{0,1} x+p_{-1,1}\right)} \pm \frac{i \sqrt{-\delta(x)}}{2\left(p_{1,1}, x^{2}+p_{0,1} x+p_{-1}, 1\right)}, x \in\left(x_{1}, x_{2}\right)\right\} .
$$

Let us take a look at $b(x)=p_{-1,0}-\left(1-p_{0,0}\right) x+p_{1,0} x^{2}$. Recall that $b(x)$ has two real roots, $x_{1}^{b}$ and $x_{2}^{b}$, such that $x_{1}<x_{1}^{b}<x_{2}<1<x_{2}^{b}$. Furthermore, $b(1)<0$ and $b(0) \geq 0$, where equality holds in the latter if and only if $p_{-1,0}=0$. Geometrically, it is easy to see that for any $x \in\left[x_{1}, x_{1}^{b}\right], b(x) \geq 0$, and therefore, the real part of the points that define $\partial D_{y}$, for $x \in\left[x_{1}, x_{1}^{b}\right]$, is non positive. As $x$ approaches $x_{1}^{b}$ in the interval $\left[x_{1}, x_{1}^{b}\right], b(x)$ continues to decrease, and therefore, the modulus

of that real part is also decreasing, on the interval $\left[x_{1}, x_{1}^{b}\right]$. Once $b(x)$ hits $x_{1}^{b}$, the real part vanishes. Therefore, $Y_{0}\left(x_{1}\right) \leq 0$ is the extreme left point on $\partial D_{y}$.

Conversely, for $x \in\left[x_{1}^{b}, x_{2}\right], b(x) \leq 0$, and therefore, the real part of the points that define $\partial D_{y}$, for $x \in\left[x_{1}^{b}, x_{2}\right]$, is non negative. Now lets take $x$ beyond the branch 
cut $\left[x_{1}, x_{2}\right]$ and see what information we can gather for $x \in\left[x_{2}, 1\right]$. We know that the image of the interval $\left[x_{2}, 1\right]$, which is on the positive real line, by either $Y_{0}(x)$ or $Y_{1}(x)$, denoted by $Y_{0}\left(\left[x_{2}, 1\right]\right)$ and $Y_{1}\left(\left[x_{2}, 1\right]\right)$, respectively, will also be intervals of $\mathbb{R}_{+}$. On account of $h(1,1)=0$, we deduce that either $Y_{0}(1)=1$ or $Y_{1}(1)=1$, which implies that 1 belongs to the image of either $Y_{0}\left(\left[x_{2}, 1\right]\right)$ or $Y_{1}\left(\left[x_{2}, 1\right]\right)$. Note that this image is a closed interval containing the point $y_{2}$, since $0<y_{2}<1$. Now take an arbitrary $y$ which belongs to the aforementioned closed image. Then both $X_{0}(x)$ and $X_{1}(x)$ are defined and we can conclude that, $Y_{0}\left(x_{2}\right)=Y_{1}\left(x_{2}\right) \geq y_{2}$ [7]. In fact, it can be shown that $Y_{0}\left(y_{2}\right)$ is at the extreme right of the contour $\partial D_{y}$. Conceptually, this indicates that the contour $\partial D_{y}$ encompasses the interval $\left[y_{1}, y_{2}\right]$

It should be noted that when a point $\varsigma \in \mathbb{C}_{x}$ approaches an $x \in\left[x_{1}, x_{2}\right]$, then $Y_{0}(\varsigma)$ approaches $y \in \partial D_{y}$ from inside $D_{y}$ and $Y_{1}(\varsigma)$ approaches $y \in \partial D_{y}$ from outside $D_{y}[5]$. Symmetrically identical results hold for $X_{ \pm}(x)$.

Remark: It is not always the case that $Y_{0}\left(x_{1}\right)<Y_{0}\left(x_{2}\right)$. However, we will assume $Y_{0}\left(x_{1}\right)<Y_{0}\left(x_{2}\right)$ throughout our analysis.

In actuality, and by definition, $Y_{ \pm}(x)$ is discontinuous on the open interval as $\left(x_{1}, x_{2}\right)$ and the value of $Y_{ \pm}(x)$ for $x \in\left(x_{1}, x_{2}\right)$ is a limiting value. As we approach $x \in\left(x_{1}, x_{2}\right)$ from above, we will use one branch, and as we approach $x \in\left(x_{1}, x_{2}\right)$ from below, we will use the other branch. Both branches will gives us different values but these values will have equal moduli, and hence the symmetric contour around the horizontal axis. The following is a more technical analysis of 
the contour produced by the branch cuts [16]:

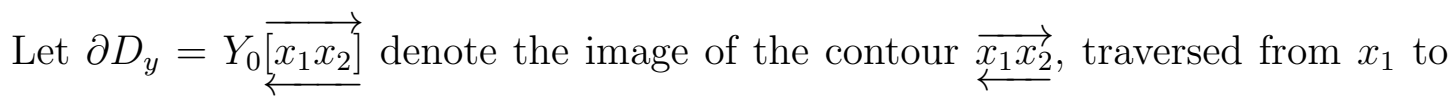
$x_{2}$ along the upper edge of the slit $\left[x_{1}, x_{2}\right]$ and then back to $x_{1}$ along the lower

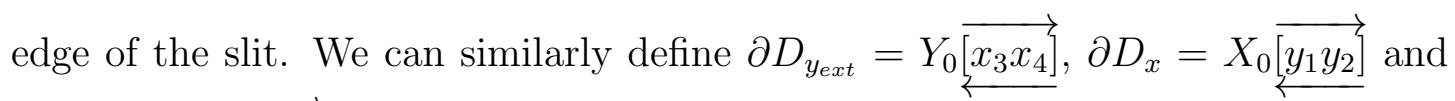

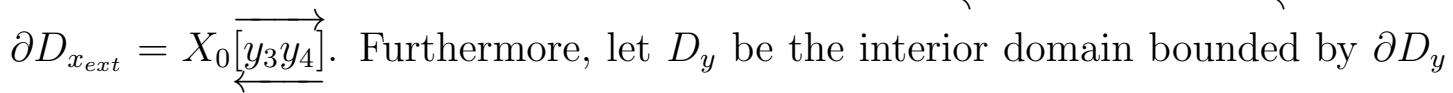
and $D_{y}^{c}$ be the exterior domain. Symmetrical meanings hold for $D_{x}$ and $D_{x}^{c}$. The following theorem, stated without proof, provides us with an important connection between the contours and branch cuts.

Define the determinant $\Delta$ as:

$$
\left|\begin{array}{ccc}
p_{1,1} & p_{1,0} & p_{1,-1} \\
p_{0,1} & p_{0,0} & p_{0,-1} \\
p_{-1,1} & p_{-1,0} & p_{-1,-1}
\end{array}\right|
$$

Theorem 3.8 [16]: For a non-singular random walk without branch points on the unit circle, we have the following properties:

1. The curves $\partial D_{y}$ and $\partial D_{y_{e x t}}$ are simple, closed, and symmetrical about the real axis in the $\mathbb{C}_{y}$ plane.

2. If $\Delta>0$, then $\left[y_{1}, y_{2}\right] \subset D_{y} \subset D_{y_{e x t}}$ and $\left[y_{3}, y_{4}\right] \subset D_{y_{\text {ext }}}{ }^{c}$.

3. If $\Delta<0$, then $\left[y_{1}, y_{2}\right] \subset D_{y_{e x t}} \subset D_{y}$ and $\left[y_{3}, y_{4}\right] \subset D_{y}^{c}$.

4. If $\Delta=0$, then $\left[y_{1}, y_{2}\right] \subset D_{y_{e x t}}=D_{y}$ and $\left[y_{3}, y_{4}\right] \subset D_{y}^{c}$.

5. $Y_{0}(x) \in D_{y} \cup D_{y_{\text {ext }}}$ for every $x$ and $X_{0}(y) \in D_{x} \cup D_{x_{e x t}}$ for every $y$. 
6. $Y_{1}(x) \in D_{y}{ }^{c} \cup D_{y_{e x t}}{ }^{c}$ for every $x$ and $X_{1}(y) \in D_{x}{ }^{c} \cup D_{x_{e x t}}{ }^{c}$ for every $y$.

Entirely symmetrical results hold for $D_{x}$ and $D_{x_{e x t}}$.

Proposition 3.9 [7]: For $x \in \mathbb{C}_{x} \backslash\left[x_{1}, x_{2}\right] \cup\left[x_{3}, x_{4}\right], X_{0}\left(Y_{0}(x)\right)=x$ and for $y \in \mathbb{C}_{y} \backslash\left[y_{1}, y_{2}\right] \cup\left[y_{3}, y_{4}\right], Y_{0}\left(X_{0}(y)\right)=y$.

Proof: Let $\mathfrak{N}(\varsigma)$ denote a neighborhood of the point $\varsigma$. For $x \in \mathfrak{N}(0)$, where $\mathfrak{N}(0)$ is sufficiently small, $Y_{0}(x)=Y_{+}(x)$ is the root with the smallest modulus such that $h(x, y)=0$. This is true since $0 \leq x_{1}<x_{1}^{b}$ and thus, $b(x) \geq 0$ for all $x \leq x_{1}$. The same property holds for $X_{0}(y)$ when $y \in \mathfrak{N}(0)$. If we take $Y_{0}(0)$, then the point 0 is the solution with the smallest modulus to the equation $h\left(0, Y_{0}(0)\right)$. Therefore, $X_{0}\left(Y_{0}(0)\right)=0$ and we can conclude that $X_{0}\left(Y_{0}(x)\right)=x$ holds for $x \in \mathfrak{N}(0)$. Since both $X_{0}(y)$ and $Y_{0}(x)$ are analytic in $\mathbb{C}_{x} \backslash\left[x_{1}, x_{2}\right] \cup\left[x_{3}, x_{4}\right]$ and $y \in \mathbb{C}_{y} \backslash\left[y_{1}, y_{2}\right] \cup\left[y_{3}, y_{4}\right]$, respectively, it follows by the Identity Theorem, (Theorem 4.5 in Chapter 4) that the equality $X_{0}\left(Y_{0}(x)\right)=x$ holds for all $x \in$ $\mathbb{C}_{x} \backslash\left[x_{1}, x_{2}\right] \cup\left[x_{3}, x_{4}\right]$. Similar arguments can be employed to prove that the equality $Y_{0}\left(X_{0}(y)\right)=y$ holds for all $y \in \mathbb{C}_{y} \backslash\left[y_{1}, y_{2}\right] \cup\left[y_{3}, y_{4}\right]$.

Proposition 3.10 [7]: $X_{0}\left(\partial D_{y}\right) \subset\left[x_{1}, x_{2}\right]$ and $Y_{0}\left(\partial D_{x}\right) \subset\left[y_{1}, y_{2}\right]$.

Proof: Let $y \in \partial D_{y}$. Since $\partial D_{y}$ is the contour image produced by $Y_{ \pm}(x)$ for $x \in\left[x_{1}, x_{2}\right]$, for any $y \in \partial D_{y}$, there exists an $x \in\left[x_{1}, x_{2}\right]$ such that, for small 
$\epsilon>0$,

$$
y=Y_{+}(x+i \epsilon), \quad \bar{y}=Y_{+}(x-i \epsilon), \quad y=Y_{-}(x-i \epsilon), \quad \bar{y}=Y_{-}(x+i \epsilon),
$$

where $\bar{y}$ denotes the complex conjugate, $x+i \epsilon$ denotes the limit of a sequence converging in the upper half plane to $x \in\left[x_{1}, x_{2}\right]$, and $x-i \epsilon$ denotes the limit of a sequence converging in the lower half plane to $x \in\left[x_{1}, x_{2}\right]$. Now, the value of $y=Y_{0}(x \pm i \epsilon)$ will depend on which branch we are required to use, and thus, will either be $Y_{+}(x \pm i \epsilon)$ or $Y_{+}(x \pm i \epsilon)$. But, by Proposition 3.9, we know that $X_{0}(y)=X_{0}\left(Y_{0}(x \pm i \epsilon)\right)=x$. Therefore $X_{0}(y) \in\left[x_{1}, x_{2}\right]$ which implies that $X_{0}\left(\partial D_{y}\right) \subset\left[x_{1}, x_{2}\right]$. 


\section{Chapter 4}

\section{Analytic Continuation}

\subsection{On the Importance of Analytic Continuation}

Insofar, we have presented a healthy portion of details and properties regarding

the functions $Y_{ \pm}(x)$ and $X_{ \pm}(y)$, which, for fixed $x$ or fixed $y$, respectively, are the solutions to the kernel equation $h(x, y)=0$ of the fundamental form. We have expressed the fact that the fundamental form is paramount to the analysis of the three generating functions of the stationary distributions for a random walk and that the kernel equation will give us a direct link between two of the generating functions. Ergo, a strong understanding of the functions $Y_{ \pm}(x)$ and $X_{ \pm}(y)$ will imply a correct understanding of how we can use the fundamental form to our benefit. Yet, we still have not provided a solid explanation as to what we will be using the fundamental form for. Our aim is not to compute or find an expression for the generating functions but rather to use the fundamental form to analytically continue the generating functions past their original domain of analyticity, which 
is the unit disk in their respective domains. We will define analytic continuation in section 4.3 , but for now, we can just crudely define analytic continuation as a method to enlarge a function's domain of analyticity. The question still remains: why do we want to analytically continue the generating functions in the first place?

We saw in section 1.4 two examples of generating functions which we were able to compute directly because we already had prior knowledge of what the probability distributions were. Even if we did not know what the probability distributions were but were given closed form expressions for the generating functions, then we could either find the probability distributions directly or get all the information required about the probability distributions through analysis of the generating functions. All this to say, if we are already given a closed form expression for the generating functions of light-tailed stationary distributions for a random walk, then our work for studying the stationary distributions becomes a smoother journey.

In reality, however, the availability of closed form solutions for the stationary distributions and generating functions is quite limited [16]. Very limited, in fact. Therefore, a significant amount of work and research has gone towards trying to find either an explicit closed form expression or an integral representation of the generating functions. This can potentially be achieved through boundary value problems. Moreover, since a closed form solution for the stationary distributions is rare, studying tail asymptotics of the distributions is crucial since they can give performance bounds and approximations. Li and Zhao showed in [16] that, when using their kernel method for exact tail asymptotics, a determination for 
the generating functions in not required when doing asymptotic analysis of the stationary distributions. In this case, all that is required is information about the dominant singularities of the generating functions, and, using a Tauberianlike theorem, we are able to obtain the exact tail asymptotic behavior for the marginal distributions and also for the joint distributions along a coordinate axis [16]. The connection between boundary value problems and the kernel method and our desire to analytically continue the generating functions is that both the boundary value problems and kernel method require the generating functions to have a larger domain of analyticity than originally prescribed.

Here are two examples that describe what is needed in terms of domain size for the generating functions. Keep in mind that our objective is not to solve the generating functions and the following examples only serve as instruments to aid in understanding the importance of analytically continuing the generating functions.

\section{Example 1: The Riemann-Hilbert Problem [7]}

To determine the generating function $\pi_{2}(y)$, say, we have the following original Riemann-Hilbert Problem:

Determine an analytic function $\pi_{2}(y)$ such that

$$
\Re\left(\frac{i h_{1}(x, y)}{h_{2}(x, y)} \pi_{2}(y)\right)=-\Re\left(\frac{i h_{0}(x, y)}{h_{2}(x, y)} \pi_{0,0}\right)
$$

for $y \in \partial D_{y}$ and $x \in\left[x_{1}, x_{2}\right]$, where $y$ equals either $Y_{+}(x)$ or $Y_{-}(x)$.

To solve the above problem, the usual method consists of considering a confor- 
mal mapping between the unit disk and the bounded domain $D_{y}$ delineated by the contour $\partial D_{y}$. The mapping can be chosen so as to preserve the symmetry with respect to the horizontal axis and by expressing the variable $x$ as a function of the variable $y$, or its conjugate $\bar{y}$, the original Riemann-Hilbert problem is transformed to a Riemann-Hilbert problem on the unit disk. For our purpose, this example demonstrates the importance of analytic continuation for $\pi_{2}(y)$. All we know is that $\pi_{2}(y)$ is analytic in the unit disk, but for the Riemann-Hilbert problem, we need $\pi_{2}(y)$ to be analytic in $D_{y}$ and, as long as $1<Y_{0}\left(x_{2}\right)$, we know that some of $D_{y}$ lies outside the unit circle. Hence, we need to analytically continue $\pi_{2}(y)$ from the unit disk to the domain $D_{y}$.

\section{Example 2: Tauberian-like Theorem for a Single Singularity [16]}

In this example, determination of an unknown generating function is not required. All that is needed is information about the location of its dominant singularity and the asymptotic property of the dominant singularity. A Tauberian-like theorem is used to link the asymptotic property of the generating function at its

dominant singularity with the tail asymptotic property of the stationary distribution that the generating function encodes. A standard Tauberian theorem requires that the probability sequence is eventually monotone, and, since our probability distributions are unknown, we can not verify that they are eventually monotone. The Tauberian-like theorem does not require monotonicity, but in exchange for this leniency, the generating function requires a larger domain of analyticity.

We first give the definition of an intended disk with radius $1+\epsilon$, denoted as 
$\Delta(\phi, \epsilon):$

For given $\epsilon>0$ and $\phi$ where $0<\phi<\frac{\pi}{2}$, the open domain $\Delta(\phi, \epsilon)$ is defined by

$$
\Delta(\phi, \epsilon)=\{z \in \mathbb{C}:|z|<1+\epsilon, z \neq 1,|z-1|>\phi\} .
$$

$A$ domain is a $\Delta$-domain at 1 if it is a $\Delta(\phi, \epsilon)$ for some $\epsilon>0$ and $0<\phi<\frac{\pi}{2}$.

\section{Tauberian-like Theorem for a Single Singularity:}

Let $A(z)=\sum_{n=0}^{\infty} a_{n} z^{n}$ be analytic at 0 with radius of convergence $R$. Suppose $R$ is a singularity of $A(z)$ on the circle of convergence such that $A(z)$ can be continued to a $\Delta$-domain at $R$. If for a real number $\alpha \notin\{0,-1,-2, \ldots\}$, $\lim _{z \rightarrow R}\left(1-\frac{1}{R}\right)^{\alpha} A(z)=g$, where $g$ is a non-zero constant, then

$$
a_{n} \sim \frac{g}{\Gamma(\alpha)} n^{\alpha-1} R^{-n}
$$

The main idea that we extract from this example is that $A(z)$ needs to be continued to a $\Delta$-domain at $R$. We will formally define the radius of convergence $R$ in the next section, but for now, we will state that the radius of convergence for both $\pi_{1}(x)$ and $\pi_{2}(y)$ is $R=1$. Hence, to use the Tauberian-like Theorem, we need to at least extend $\pi_{1}(x)$ and $\pi_{2}(y)$ to a $\Delta$-domain at 1 , which is an intended disk. This extension is again achieved via analytic continuation.

The two preceding examples emphasize the need for analytic continuation of the generating functions $\pi_{1}(x)$ and $\pi_{2}(y)$, when these generating functions are unknown to us. The next section introduces power series and gives needed results on power series. 


\subsection{Power Series \& Analytic Continuation}

Recall that a power series is a series of the form $\sum_{n=0}^{\infty} a_{n}\left(z-z_{0}\right)^{n}$ where $z_{0}, a_{n}$ $\in \mathbb{C}$ for any $n \in \mathbb{N}_{0}$ and $z$ is any complex number in the stated domain. Note that a generating function is a power series. The radius of convergence of the power series $\sum_{n=0}^{\infty} a_{n}\left(z-z_{0}\right)^{n}$ is denoted and defined by $R=\sup \left\{|z|: \sum_{n=0}^{\infty} \mid a_{n}(z-\right.$ $\left.z_{0}\right)^{n} \mid$ converges $\}$. The series is absolutely convergent for all $z$ belonging to the open disk centered at $z_{0}$ with radius $R$, denoted by $\mathcal{D}_{z}\left(z_{0}, R\right)$ and divergent for all $z$ outside the closure of $\mathcal{D}_{z}\left(z_{0}, R\right)$. The boundary of $\mathcal{D}_{z}\left(z_{0}, R\right)$ is a circle called the circle of convergence. Note that $R$ need not be finite. The following theorem solidifies the preceding discussion.

Theorem 4.1 [8]: For every generating function $A(z)=\sum_{n=0}^{\infty} a_{n} z^{n}$ with radius of convergence $0 \leq R \leq \infty$, we have:

1. $\sum_{n=0}^{\infty} a_{n} z^{n}$ converges absolutely for $|z|<R$.

2. $\sum_{n=0}^{\infty} a_{n} z^{n}$ diverges for $|z|>R$.

3. There exists an $v<R$ such that $\sum_{n=0}^{\infty} a_{n} z^{n}$ converges uniformly for $|z| \leq v$.

Proof: Omitted

Also recall that a complex valued function $f(z)$ is analytic at a point $z_{0} \in \mathbb{C}$ if it is differentiable at $z_{0}$ and at each point in some neighborhood of $z_{0}$ [12]. The existence of a complex derivative is a powerful attribute for a function to posses, for it implies that any analytic function is infinitely differentiable and 
equal to its own Taylor series [12], which is a power series whose coefficients can be completely determined. Indeed, we have the following result: a complex valued function $f(z)$ is analytic in an open disk $U \in \mathbb{C}$, centered at $z_{0}$ if and only if its Taylor series converges to the value of the function for every point $z \in U$ [12]. This result can be extended to a function of several complex variables: a functions $f(z) \equiv f\left(z_{1}, \ldots, z_{n}\right)$ is analytic on a domain $U \subset \mathbb{C}^{n}$ if for each point $a=\left(a_{1}, \ldots, a_{n}\right) \in U, f(z)$ can be expressed as a power series expansion $\sum_{k 1, \ldots, k_{n}}\left(z^{1}-a^{1}\right) \ldots\left(z^{n}-a^{n}\right)^{k_{n}}$ [12]. Another important definition is that of a meromorphic function which is a function that is analytic in some region, except possibly at poles [12].

At this point, we shall present useful theorems that we will need when describing the properties of the generating functions and analytically continuing the generating functions for the random walk.

Remark: In what follows we assume, without the lose of generality, that $z_{0}=0$ so that we have the power series $\sum_{n=0}^{\infty} a_{n} z^{n}$.

Theorem 4.2 [8] : If $\sum_{n=0}^{\infty} a_{n} z^{n}$ has a radius of convergence $R<\infty$ then $f(z)=$ $\sum_{n=0}^{\infty} a_{n} z^{n}$ has at least one singularity on the circle $|z|=R$.

Proof: Suppose that the power series does not have a singularity on its circle of convergence $|z|=R$. Then $f(z)$ is analytic for every $z$ such that $|z|=R$ and therefore $f(z)$ has a power series expansion around each $z$. This implies that for each $z$ such that $|z|=R$, there exists an $\epsilon_{z}$, dependent on $z$, such that $f(z)$ is analytic in $\mathcal{D}_{z}\left(z, \epsilon_{z}\right)$. Since the circle is compact, we can take $\min _{|z|=R} \epsilon_{z}=\epsilon>0$ 
and hence, there exists a function $g(z)$ analytic in $\mathcal{D}_{z}(z, R+\epsilon)$ such that $g(z)=$ $f(z)$ in $\mathcal{D}_{z}(0, R)$. By the analyticity of $g(z), g(z)$ has a power series representation $\sum_{n=0}^{\infty} b_{n} z^{n}$ which is convergent for all $z$ such that $|z|<R+\epsilon$. However, $g(z)=$ $f(z)=\sum_{n=0}^{\infty} a_{n} z^{n}$ for all $|z|<R$, so, by the uniqueness theorem, $a_{n} \equiv b_{n}$. But then the radius of convergence would be $R$, so we have a contradiction.

Theorem 4.3 [8]: If $\sum_{n=0}^{\infty} a_{n} z^{n}$ has a radius of convergence $R<\infty$ and $a_{n} \geq 0$ for all $n$, then $f(z)=\sum_{n=0}^{\infty} a_{n} z^{n}$ has a singularity at $z=R$.

Proof: By the above proposition, $f(z)$ has a singularity on $|z|=R$, say the point, written in polar form, $R e^{i \alpha}$. Now consider a power series for $f(z)$ about a point inside the circle $|z|=R$, say $\rho e^{i \alpha}$, so that $0<\rho<R$. Then $f(z)=$ $\sum_{n=0}^{\infty} \frac{f(z)^{(n)}\left(\rho e^{i \alpha}\right)}{n !}\left(z-\rho e^{i \alpha}\right)^{n}$ and the radius of convergence for this power series is $R-\rho$. Visually we can see that the radius of convergence cannot be bigger than $R-\rho$ since otherwise the power series would extend $f(z)$ analytically beyond $R e^{i \alpha}$. Also notice that for any $j \in \mathbb{N}_{0}$,

$$
f(z)^{(j)}\left(\rho e^{i \alpha)}=\sum_{n=j}^{\infty} a_{n} n(n-1) \ldots(n-j+1)\left(\rho e^{i \alpha}\right)^{n-j} .\right.
$$

Since $a_{n} \geq 0$, we have $\mid f(z)^{(j)}\left(\rho e^{i \alpha)} \mid \leq f(z)^{(j)}(\rho)\right.$. Therefore, the power series expansion of $f(z)$ about $\rho$,

$$
\sum_{n=0}^{\infty} \frac{f(z)^{(n)}(\rho)}{n !}(z-\rho)^{n}
$$

must have a radius of convergence $R-\rho$. On the other hand, if $f(z)$ were analytic at the point $z=R$, then the above power series would converge in a disc with radius greater than $R-\rho$. Therefore, $f(z)$ must have a singular point at $z=R$. 
We have spoken quite a bit about convergence, but we have yet to mention continuity. Suppose we take a $b$, say, such that $|b|<v$. Then, by Theorem 4.1 (3), that $\sum_{n=0}^{\infty} a_{n} z^{n}$ converges uniformly for $|z| \leq v$, and, since each $z^{n}$ is continuous at $b$, for every $n$, we have:

$$
\lim _{z \rightarrow b} A(z)=\lim _{z \rightarrow b} \sum_{n=0}^{\infty} a_{n} z^{n}=\sum_{n=0}^{\infty} \lim _{z \rightarrow b} a_{n} z^{n}=A(b)<\infty .
$$

Therefore, $A(b)$ is continuous at $b$, for $|b|<v$.

However, this result does not give us information regarding the behavior of $A(z)$ as $z \rightarrow R^{-}$. Abel's Theorem rectifies this situation [8]. Without loss of generality we assume $R=1$.

Theorem 4.4 Abel's Convergence Theorem [8]: Let $A(z)=\sum_{n=0}^{\infty} a_{n} z^{n}$ with $|z|<1$. Then,

$$
\text { if } \sum_{n=0}^{\infty} a_{n}=a<\infty, \text { then } \lim _{z \rightarrow 1^{-}} A(z)=a .
$$

Proof: Omitted

Remark: The converse of Abel's Theorem is also true [8].

Let us look at our generating function $\pi_{1}(x)=\sum_{m=1}^{\infty} \pi_{m, 0} x^{m-1}$. Clearly, $\left\{\pi_{m, 0}\right\}$ is bounded by 1 and since $\left|\pi_{1}(x)\right| \leq \frac{1}{1-|x|}$ we know that $\pi_{1}(x)$ converges absolutely for at least $|x|<1$ and diverges for $|x|>1$. Moreover, by Abel's Theorem, since $\sum_{m=0}^{\infty} \pi_{m, 0}=1$, we have $\lim _{z \rightarrow 1^{-}} \pi_{1}(x)=1$. So we can state the 
following, with absolute certainty:

1. $\pi_{1}(x)$ is analytic in the open domain $\left\{x \in \mathbb{C}_{x}:|x|<1\right\}$,

2. $\pi_{1}(x)$ is continuous in the closed domain $\left\{x \in \mathbb{C}_{x}:|x|<1\right\}$.

But wait! We forgot a very important piece when claiming the analyticity of $\pi_{1}(x)$ in the unit disk. What about the branch cut $\left[x_{1}, x_{2}\right]$ ? To which we reply: no problem. There is a result in complex analysis, which we will use, and state without proof, to show that $\pi_{1}(x)$ is analytic throughout the unit disk, branch cut included: If a function $f(z)$ is continuous in a domain $D$ and is analytic in $D \backslash \mathcal{C}$, where $\mathcal{C}$ is an open curve inside $D$ then $f(z)$ is analytic in the whole domain $D$ [17.

The same results hold for both $\pi_{2}(y)$ and $\pi(x, y)$, in their respective domains. Therefore, all three generating functions have a radius of convergence $R=1$. Again, we assume that the generating functions are unknown to us and we have already seen the methods required to either solve the generating functions or analyze their asymptotic behavior. Whatever the chosen direction is, however, all techniques require a larger radius of convergence for the generating functions and what follows is a more formal definition of analytic continuation.

When given an analytic function $f_{1}(z)$, say, defined in a region $\mathfrak{R}_{1}$ of $\mathbb{C}$, it is desirable, as in our case, to know if the domain of analyticity can be extended. That is, we would like to know if there exists an analytic function $f(z)$, in a larger region $\mathfrak{R}$, with $\mathfrak{R}_{1} \subset \mathfrak{R}$, such that $f(z)=f_{1}(z)$ for all $z \in \mathfrak{R}_{1}$. The following is a 
simple example to illustrate analytic continuation:

\section{Example}

Consider the function $f(z)$, for $z \in \mathbb{C}$ defined as

$$
f(z)= \begin{cases}\sum_{n=0}^{\infty} z^{n}, & \text { if }|z|<1 \\ \frac{1}{1-z}, & \text { if } z \neq 0 .\end{cases}
$$

We recognize $\sum_{n=0}^{\infty} z^{n}$ as a geometric series and we know that is converges for all $|z|<1$. Note that this series is a power series and, as such, a Taylor series for an analytic function. In fact, for any $|z|<1$, the series converges to $\frac{1}{1-z}$. That is, $\sum_{n=0}^{\infty} z^{n}=\frac{1}{1-z}$ for $|z|<1$. Notice $\sum_{n=0}^{\infty} z^{n}$ diverges for $|z| \geq 1$ but $\frac{1}{1-z}$ is analytic everywhere in $\mathbb{C}$ except at the point $z \neq 1$. We call $\frac{1}{1-z}$ the analytic continuation of $\sum_{n=0}^{\infty} z^{n}$. In a sense, this example explains the notion of analytic continuation in a clear and intuitive way. We have a representation of a function as a power series, namely $\sum_{n=0}^{\infty} z^{n}$, which is analytic in a certain region $(|z|<1)$, and then we found another representation, $\frac{1}{1-z}$, which matches the first representation, point by point, in the first region of analyticity, yet $\frac{1}{1-z}$ is analytic in another region, which is bigger, and includes the first region, $|z|<1$.

The following is a formal definition of analytic continuation [12]:

Let $f_{1}(z)$ be analytic in a region $\mathfrak{R}_{1} \subset \mathbb{C}$. Let $\mathfrak{R}_{2} \subset \mathbb{C}$ be another region such at $\mathfrak{R}_{1} \cap \mathfrak{R}_{2}=\mathfrak{R} \neq \varnothing$. If there exists a function $f_{2}(z)$ analytic on $\mathfrak{R}_{2}$, with 
$f_{1}(z)=f_{2}(z)$ for all $z \in \mathfrak{R}$ then $f_{2}(z)$ is an analytic continuation of $f_{2}(z)$ to $\mathfrak{R}_{2}$.

Note that, if we define

$$
f(z)=\left\{\begin{array}{ll}
f_{1}(z), & \text { if } z \in \mathfrak{R}_{1} \\
f_{2}(z), & \text { if } z \in \mathfrak{R}_{2}
\end{array},\right.
$$

then $f(z)$ is analytic in $\mathfrak{R}_{1} \cup \mathfrak{R}_{2}$.

There are a variety of ways to analytically continue an analytic function, when possible, including the simple method of using the function's own Taylor series and expanding around various points inside the circle of convergence [12]. Our technique will involve using the fundamental form and the Identity Theorem, which will be stated without proof.

Theorem 4.5 Identity Theorem [12]: If $f(z)$ and $g(z)$ are analytic on some domain $D$ in $\mathbb{C}_{z}$ and if $f(z)=g(z)$ for all $z \in U \subset D$ that has a limit point in $D$ then $f(z)=g(z)$ for all $z \in D$.

Once again, we will assume that we have a stable, non-singular and light-tailed random walk. Now let us revisit the fundamental form,

$$
-h(x, y) \pi(x, y)=h_{1}(x, y) \pi_{1}(x)+h_{2}(x, y) \pi_{2}(y)+h_{0}(x, y) \pi_{0,0},
$$

along with the algebraic curve $\mathcal{B}=\left\{(x, y) \in \mathbb{C}^{2}: h(x, y)=0\right\}$.

We already know that $h(x, y)=0$ gives two roots in terms of $x$ and two roots in terms of $y$, and we have attained enough knowledge, for our benefit, about these 
roots, so we can safely speak of $h\left(x, Y_{0}(x)\right)=0$.

We want to set the left hand side of the fundamental form to 0 which will consequently enable us to play with the two generating functions $\pi_{1}(x)$ and $\pi_{2}(y)$. However, $h\left(x, Y_{0}(x)\right)=0$ will not suffice in making the left hand side vanish, as we also require the additional stipulation that $\left|\pi\left(x, Y_{0}(x)\right)\right|<\infty$. This issue can be easily mended since, as previously shown, $Y_{0}(x)$ is analytic on the unit circle $|x|=1$ and $Y_{0}(x) \leq 1$ on the unit circle $|x|=1$. Since $\pi(x, y)$ is analytic in the open domain $\left\{(x, y) \in \mathbb{C}^{2}:|x|<1,|y|<1\right\}$ and continuous in the closed domain $\left\{(x, y) \in \mathbb{C}^{2}:|x| \leq 1,|y| \leq 1\right\}, \pi\left(x, Y_{0}(x)\right)<\infty$ for $|x| \leq 1$.

Now we have the new equation, for $|x| \leq 1$,

$$
h_{1}\left(x, Y_{0}(x)\right) \pi_{1}(x)+h_{2}\left(x, Y_{0}(x)\right) \pi_{2}\left(Y_{0}(x)\right)+h_{0}\left(x, Y_{0}(x)\right) \pi_{0,0}=0 .
$$

with both $\pi_{1}(x)$ and $\pi_{2}\left(Y_{0}(x)\right)$ still well defined. Our aim is to analytically continue $\pi_{2}(y)$ past its radius of convergence, which is $R=1$, without using advanced mathematics and just restricting ourselves to the information we have gathered insofar. For our purpose, we would like to use (4.1) and isolate either $\pi_{1}(x)$ and $\pi_{2}(y)$ to the left hand side of (4.1) and then determine the region of analyticity of the right hand side, all the while ensuring that the generating function on the right hand side of (4.1) is finite. Although there is no clear cut algorithm, we will analysis two different models in the next two sections and see if we can conclude some sort of pattern or extract some information that may be implemented for a general model. We conclude this section by reiterating facts and results which we have shown to be true and which we will need for the following sections: 
1. $h_{k}$ and $H_{k}$, for $k=0,1,2$, are all polynomials and are analytic everywhere in their respective complex planes.

2. $Y_{0}(x)$ and $X_{0}$ are analytic in their respective cut planes.

3. $\pi_{1}(x)$ and $\Pi_{1}(x)$ are analytic for all $|x|<1$ and continuous for all $|x| \leq 1$.

4. $\pi_{2}(y)$ and $\Pi_{2}(y)$ are analytic for all $|y|<1$ and continuous for all $|y| \leq 1$.

5. $\left|Y_{0}(x)\right| \leq 1$ and $\left|X_{0}(y)\right| \leq 1$ for $|x|=1$ and $|y|=1$, respectively.

6. $Y_{0}(x) \in D_{y} \cup D_{y_{e x t}}$ for every $x$ and $X_{0}(y) \in D_{x} \cup D_{x_{e x t}}$ for every $y$.

7. By the Maximum Modulus Principle, $\left|Y_{0}(x)\right|$ and $\left|X_{0}(y)\right|$ attain their maximum value on the boundary of $\left[x_{1}, x_{2}\right]$ and $\left[y_{1}, y_{2}\right]$, respectively [12].

Note: Item 7 mentions the very useful Maximum Modulus Principle [12]: if $f(z)$ is analytic inside a simple closed contour $\mathcal{C}$ and continuous on $\mathcal{C}$, and is not identically constant, then the maximum value of $|f(z)|$, inside and on $\mathcal{C}$, occurs on $\mathcal{C}$. 


\subsection{Two Coupled Processor Model}

This example is the discrete-time version of the first three sections found in Guy Fayolle's and Roudolf Iasnogorodski's paper, Two Coupled Processors: The Reduction to a Riemann-Hilbert Problem [3]. The queueing system considered, which was modeled and analyzed as a continuous-time two-dimensional Markov chain, consists of two parallel $M / M / 1$ queues, $Q_{1}$ and $Q_{2}$, both with infinite capacities. The arrivals form two independent Poisson processes with parameters $\lambda_{1}$ and $\lambda_{2}$, for $Q_{1}$ and $Q_{2}$, respectively. The service discipline is first-in first-out for each queue and the service times are exponentially distributed with instantaneous service rates $S_{1}$ and $S_{2}$, for $Q_{1}$ and $Q_{2}$, respectively. Furthermore, we define $S_{1}$ and $S_{2}$ according to the size of each queue: if both $Q_{1}$ and $Q_{2}$ are non empty, then $S_{1}=\mu_{1}$ and $S_{2}=\mu_{2}$, if $Q_{2}$ is empty, then $S_{1}=\mu_{1}^{*}$, and if $Q_{2}$ is empty, then $S_{2}=\mu_{2}^{*}$.

We let $\lambda_{1}+\lambda_{2}+\mu_{1}+\mu_{2}=1$ and, upon uniformization, we obtain a random walk in the quarter plane, with transition probabilities:

$$
\begin{array}{llll}
p_{0,1}=\lambda_{2} & p_{1,0}=\lambda_{1} & p_{0,-1}=\mu_{2} & p_{-1,0}=\mu_{1} \\
p_{0,1}^{(1)}=\lambda_{2} & p_{1,0}^{(1)}=\lambda_{1} & p_{-1,0}^{(1)}=\mu_{1}^{*} & p_{0,0}^{(1)}=1-\left(\lambda_{2}+\lambda_{1}+\mu_{1}^{*}\right) \\
p_{0,1}^{(2)}=\lambda_{2} & p_{1,0}^{(2)}=\lambda_{1} & p_{0,-1}^{(2)}=\mu_{2}^{*} & p_{0,0}^{(2)}=1-\left(\lambda_{2}+\lambda_{1}+\mu_{2}^{*}\right) \\
p_{0,1}^{(0)}=\lambda_{2} & p_{1,0}^{(0)}=\lambda_{1} & p_{0,0}^{(0)}=\mu_{2}+\mu_{1} .
\end{array}
$$


We can further define our functions for the fundamental form as:

$$
\begin{array}{lll}
a(x)=\lambda_{2} x & b(x)=\mu_{1}-x+\lambda_{1} x^{2} & c(x)=\mu_{2} x \\
\tilde{a}(y)=\lambda_{1} & \tilde{b}(y)=\mu_{2}-y+\lambda_{2} y^{2} & \tilde{c}(y)=\mu_{1} y \\
a_{1}(x)=\lambda_{2} x & b_{1}(x)=\mu_{1}^{*}-\left(\lambda_{1}+\lambda_{2}+\mu_{1}^{*}\right) x+\lambda_{1} x^{2} & \\
\tilde{a}_{1}(y)=\lambda_{1} & \tilde{b}_{1}(y)=-\left(\lambda_{1}+\lambda_{2}+\mu_{1}^{*}\right)+\lambda_{2} y^{2} & \tilde{c}_{1}(y)=\mu_{1}^{*} \\
a_{2}(x)=\lambda_{2} & b_{2}(x)=\lambda_{1} x-\left(\lambda_{1}+\lambda_{2}+\mu_{2}^{*}\right) & \\
\tilde{a}_{2}(y)=\lambda_{1} y & \tilde{b}_{2}(y)=\mu_{2}^{*}-\left(\lambda_{1}+\lambda_{2}+\mu_{2}^{*}\right) y+\lambda_{2} y^{2} & \\
a_{0}(x)=\lambda_{2} & b_{0}(x)=\lambda_{1} x+\mu_{1}+\mu_{2}-1 & \\
\tilde{a}_{0}(y)=\lambda_{1} & \tilde{b}_{0}(y)=\lambda_{2} y+\mu_{1}+\mu_{2}-1 &
\end{array}
$$

Since $\mathbf{M} \neq 0$, we can use Theorem 2.2 to establish stability conditions for the system:

$$
\begin{aligned}
& M_{y}=\lambda_{2}-\mu_{2}<0 \\
& M_{x}=\lambda_{1}-\mu_{1}<0 \\
& M_{x} M_{y}^{(1)}-M_{y} M_{x}^{(1)}=\left(\lambda_{1}-\mu_{1}\right)\left(\lambda_{2}\right)-\left(\lambda_{2}-\mu_{2}\right)\left(\lambda_{1}-\mu_{1}^{*}\right)<0 \\
& M_{y} M_{x}^{(2)}-M_{x} M_{y}^{(2)}=\left(\lambda_{2}-\mu_{2}\right)\left(\lambda_{1}\right)-\left(\lambda_{1}-\mu_{1}\right)\left(\lambda_{2}-\mu_{2}^{*}\right)<0 .
\end{aligned}
$$

From the last two equalities, we get: 


$$
\left(\lambda_{1}-\mu_{1}\right)\left(\lambda_{2}\right)<\left(\lambda_{2}-\mu_{2}\right)\left(\lambda_{1}-\mu_{1}^{*}\right) \text { and }\left(\lambda_{2}-\mu_{2}\right)\left(\lambda_{1}\right)<\left(\lambda_{1}-\mu_{1}\right)\left(\lambda_{2}-\mu_{2}^{*}\right),
$$

which gives us:

$$
\begin{aligned}
\frac{\left(\lambda_{2}-\mu_{2}\right)}{\left(\lambda_{2}-\mu_{2}^{*}\right)} \cdot \lambda_{1}<\left(\lambda_{1}-\mu_{1}\right) \cdot \frac{\lambda_{2}}{\lambda_{2}} & \Longrightarrow \quad \lambda_{1} \lambda_{2}<\left(\lambda_{2}-\mu_{2}^{*}\right)\left(\lambda_{1}-\mu_{1}^{*}\right) \\
& \Longrightarrow 0<\mu_{1}^{*} \mu_{2}^{*}-\mu_{1}^{*} \lambda_{2}-\mu_{2}^{*} \lambda_{1} \\
& \Longrightarrow 0<1-\frac{\lambda_{1}}{\mu_{1}^{*}}-\frac{\lambda_{2}}{\mu_{2}^{*}}
\end{aligned}
$$

Therefore, the random walk is ergodic if $1-\frac{\lambda_{1}}{u_{1}^{*}}-\frac{\lambda_{2}}{u_{2}^{*}}>0$. Henceforth, we assume the ergodicity condition holds.

Now we can define our kernel function to be:

$$
h(x, y)=y^{2}\left(\lambda_{2} x\right)+\left(\lambda_{1} x^{2}-x+\mu_{1}\right) y+\mu_{2} x
$$

and the roots of the kernel equations $h(x, y)=0$, for fixed $x$, to be:

$$
\begin{aligned}
Y_{ \pm}(x) & =\frac{\left(x-\mu_{1}-\lambda_{1} x^{2}\right) \pm \sqrt{\left(\lambda_{1} x^{2}-x-\mu_{1}\right)^{2}-4 \lambda_{2} \mu_{2} x^{2}}}{2 \mu_{2} x} \\
& =\frac{x\left(1-\lambda_{1} x-\frac{\mu_{1}}{x}\right) \pm \sqrt{x^{2}\left[\left(\lambda_{1} x-1+\frac{\mu_{1}}{x}\right)-4 \lambda_{2} \mu_{2}\right]}}{2 \mu_{2} x} \\
& =\frac{\left(1-\lambda_{1} x-\frac{\mu_{1}}{x}\right) \pm \sqrt{\left(\lambda_{1} x-1+\frac{\mu_{1}}{x}\right)^{2}-4 \lambda_{2} \mu_{2}}}{2 \mu_{2}}
\end{aligned}
$$

Since $p_{1,1}=p_{1,-1}=p_{-1,1}=p_{-1,-1}=0, p_{1,0}=\lambda_{1}$ and $p_{-1,0}=\mu_{1}$, by Theorem 
3.1 and Corollary 3.2, $Y_{ \pm}(x)$ has four real branch points with $0<x_{1}<x_{2}<1<$ $x_{3}<x_{4}<\infty$. Accordingly, we make our branch cuts, in the $\mathbb{C}_{x}$ plane, to be the two intervals $\left[x_{1}, x_{2}\right]$ and $\left[x_{3}, x_{4}\right]$.

Again, we will denote $Y_{0}(x)$ to be that branch of $Y_{ \pm}(x)$ with the smaller modulus and $X_{0}(y)$ to be that branch of $X_{ \pm}(y)$ with the smaller modulus. We will also use the following curves:

$\mathfrak{Y}=\left\{Y_{0}(x):|x|=1\right\}$. It is simple, closed, and lies within $\mathcal{D}_{y}(0,1)$.

$\mathfrak{X}=\left\{X_{0}(y):|y|=1\right\}$. It is simple, closed, and lies within $\mathcal{D}_{x}(0,1)$.

$\mathfrak{Y}^{\prime}=\frac{\mu_{2}}{\lambda_{2} \mathfrak{Y}}$. It is simple, closed, and lies outside $\overline{\mathcal{D}}_{y}(0,1)$.

$\mathfrak{X}^{\prime}=\frac{\mu_{1}}{\lambda_{1} \mathfrak{X}}$. It is simple, closed, and lies outside $\overline{\mathcal{D}}_{x}(0,1)$.

We note that both $\mathfrak{Y}^{\prime}$ and $\mathfrak{X}^{\prime}$ are the curves obtained from $\mathfrak{Y}$ and $\mathfrak{X}$ under the conformal mappings

$z \rightarrow \frac{\mu_{2}}{\lambda_{2} z}$ and $z \rightarrow \frac{\mu_{1}}{\lambda_{1} z}$ respectively.

Note: It should be noted that the roots $x_{1}, x_{2}, x_{3}$ and $x_{4}$ were determined, where

$$
x_{1}=x_{1}\left(\lambda_{1}, \lambda_{2}, \mu_{1}, \mu_{2}\right), x_{2}=x_{2}\left(\lambda_{1}, \lambda_{2}, \mu_{1}, \mu_{2}\right), x_{3}=\frac{\mu_{1}}{\lambda_{1} x_{2}} \text { and } x_{4}=\frac{\mu_{1}}{\lambda_{1} x_{1}} .
$$

To continue, we want to obtain as much information as we can about the roots $Y_{0}(x)$ and $X_{0}(y)$. Using all of our previous results, we are able to list the following useful properties belonging to $Y_{0}(x)$ : 
1. The equation $h(x, y)=0$ has one root $Y_{0}(x)$ inside the unit circle which is an analytic function of $x$ in the whole complex plane, cut along the two segments $\left[x_{1}, x_{2}\right]$ and $\left[x_{3}, x_{4}\right]$.

2. $\left|Y_{0}(x)\right|=\sqrt{\frac{\mu_{2}}{\lambda_{2}}} \quad$ if $x \in\left[x_{1}, x_{2}\right] \cup\left[x_{3}, x_{4}\right]$.

3. $\left|Y_{0}(x)\right| \leq \sqrt{\frac{\mu_{2}}{\lambda_{2}}} \quad \forall x \in \mathbb{C}_{x}$.

4. $\left|Y_{0}(x)\right| \leq 1$ for $|x|=1$.

5. $Y_{0}(1)=\min \left(1, \frac{\mu_{2}}{\lambda_{2}}\right)$.

6. $Y_{1}(x)=\frac{\mu_{2}}{\lambda_{2} Y_{0}(x)}$ is the second root of $h(x, y)=0$ and when $x \in\left[x_{1}, x_{2}\right] \cup$ $\left[x_{3}, x_{4}\right], Y_{0}(x)$ and $Y_{1}(x)$ are complex conjugates with a common modules equal to $\sqrt{\frac{\mu_{2}}{\lambda_{2}}}$.

Similarly, for $X_{0}(y)$, we have:

1. The equation $h(x, y)=0$ has one root $(y)$ inside the unit circle which is an analytic function of $y$ in the whole complex plane, cut along the two segments $\left[y_{1}, y_{2}\right]$ and $\left[y_{3}, y_{4}\right]$.

2. $\left|X_{0}(y)\right|=\sqrt{\frac{\mu_{1}}{\lambda_{1}}} \quad$ if $y \in\left[y_{1}, y_{2}\right] \cup\left[y_{3}, y_{4}\right]$.

3. $\left|X_{0}(y)\right| \leq \sqrt{\frac{\mu_{1}}{\lambda_{1}}} \quad \forall y \in \mathbb{C}_{y}$.

4. $\left|X_{0}(y)\right| \leq 1$ for $|y|=1$. 
5. $X_{0}(1)=\min \left(1, \frac{\mu_{1}}{\lambda_{1}}\right)$.

6. $X_{1}(y)=\frac{\mu_{1}}{\lambda_{1} X_{0}(y)}$ is the second root of $h(x, y)=0$ and when $y \in\left[y_{1}, y_{2}\right] \cup$ $\left[y_{3}, y_{4}\right], X_{0}(y)$ and $X_{1}(y)$ are complex conjugates with a common modules equal to $\sqrt{\frac{\mu_{1}}{\lambda_{1}}}$.

Remark: Note that the contours $\partial D_{x}$ and $\partial D_{y}$ are simply circles, $|x|=\frac{\mu_{1}}{\lambda_{1}}$ and $|y|=\frac{\mu_{2}}{\lambda_{2}}$, respectively. This fact makes the analysis significantly less complicated.

For item 1 from the first list above, the fact that $h(x, y)=0$ has only one root in the unit disk can be shown using Rouché's Theorem [12]: if $f(z)$ and $g(z)$ are analytic inside and on a simple closed contour $\mathcal{C}$ and $|g(z)|<|f(z)|$ on $\mathcal{C}$ then $f(z)$ and $f(z)+g(z)$ have the same number of roots inside $\mathcal{C}$. On the circle $|y|=1$ we have $|x|\left|y^{2} \lambda_{2}+\mu_{2}\right| \leq|x y|\left|\lambda_{1} x-1+\frac{\mu_{1}}{x}\right|$, and we see that for $|x|=1$ and $x \neq 1$, $h(x, y)=0$ has exactly in root inside the unit circle.

Moreover, the claim that $\left|Y_{0}(x)\right|=\sqrt{\frac{\mu_{2}}{\lambda_{2}}} \quad$ if $x \in\left[x_{1}, x_{2}\right] \cup\left[x_{3}, x_{4}\right]$ follows directly from the Maximum Modulus Principle. Since the maximum value of $\left|Y_{0}(x)\right|$ can only be reached on the boundary of $\left[x_{1}, x_{2}\right] \cup\left[x_{3}, x_{4}\right]$ we have, for $x \in\left(x_{1}, x_{2}\right) \cup\left(x_{3}, x_{4}\right):$

$$
\begin{aligned}
& Y_{0}(x)=\frac{\left(1-\lambda_{1} x-\frac{\mu_{1}}{x}\right)-i \sqrt{-\left(\lambda_{1} x-1+\frac{\mu_{1}}{x}\right)^{2}+4 \lambda_{2} \mu_{2}}}{2 \mu_{2}}, \text { which gives us, } \\
& \left|Y_{0}(x)\right|=\sqrt{\Re\left[Y_{0}(x)\right]+\Im\left[Y_{0}(x)\right]}=\sqrt{\frac{\mu_{2}}{\lambda_{2}}} .
\end{aligned}
$$

At this point, we know that the closed contours produced by the branches along the cuts inside the unit disks are circles with center 0. But we still have 
not established if the radii of these circles are smaller or larger than 1. Four cases arise when dealing with the various regions that $Y_{0}(x)$ and $X_{0}(y)$ produce:

1. If $\frac{\mu_{2}}{\lambda_{2}}<1$, then $\frac{\mu_{2}}{\lambda_{2}}<\sqrt{\frac{\mu_{2}}{\lambda_{2}}}<1, Y_{0}(1)=\frac{\mu_{2}}{\lambda_{2}}$ and $|\mathfrak{Y}| \leq \sqrt{\frac{\mu_{2}}{\lambda_{2}}}$.

In fact, the region $\mathcal{D}_{x}(0,1)$ is mapped by $Y_{0}(x)$ onto a ring shaped region in $\mathbb{C}_{y}$, where the outer curve is the circle $|y|=\sqrt{\frac{\mu_{2}}{\lambda_{2}}}$ and the inner curve is $\mathfrak{Y}$. We will denote this ring shaped region as $G_{y}$. Note that $G_{y} \subset \mathcal{D}_{x}(0,1)$ and the point $y=1 \in \mathfrak{Y}^{\prime}$.

2. If $\frac{\mu_{2}}{\lambda_{2}}>1$, then $\frac{\mu_{2}}{\lambda_{2}}>\sqrt{\frac{\mu_{2}}{\lambda_{2}}}>1, Y_{0}(1)=1$ and $|\mathfrak{Y}| \leq 1$.

Again, as in the first case, the region $\mathcal{D}_{x}(0,1)$ is mapped by $Y_{0}(x)$ onto a ring shaped region in $\mathbb{C}_{y}$, where the outer curve is the circle $|y|=\sqrt{\frac{\mu_{2}}{\lambda_{2}}}$ and the inner curve is $\mathfrak{Y}$. However, for this ring shaped region, $\mathcal{D}_{y}(0,1) \subset|y|=\sqrt{\frac{\mu_{2}}{\lambda_{2}}}$ and the curve $\mathfrak{Y}$ is has a value of at most 1 , since $Y_{0}(1)=1$ and the rest of the curve either lies on, or within, $\mathcal{D}_{y}(0,1)$. We will denote the region between the unit circle $|y|=1$ and $\mathfrak{Y}$ as $R_{y}$.

3. If $\frac{\mu_{1}}{\lambda_{1}}<1$, then $\frac{\mu_{1}}{\lambda_{1}}<\sqrt{\frac{\mu_{1}}{\lambda_{1}}}<1, X_{0}(1)=\frac{\mu_{1}}{\lambda_{1}},|\mathfrak{X}| \leq \sqrt{\frac{\mu_{1}}{\lambda_{1}}}$, and the point $x=1 \in$ $\mathfrak{X}^{\prime}$. A parallel ring shaped region as presented in the first case can be drawn for this case and symmetric results follow.

4. If $\frac{\mu_{1}}{\lambda_{1}}>1$ then $\frac{\mu_{1}}{\lambda_{1}}>\sqrt{\frac{\mu_{1}}{\lambda_{1}}}>1, X_{0}(1)=1$ and $|\mathfrak{X}| \leq 1$. A parallel ring shaped region as presented in the second case can be drawn for this case and symmetric 
results follow.

We further define $R_{y}$ to be the region between the unit circle $|y|=1$ and the curve $\mathfrak{Y}$. Also, we let $R_{x}$ be the region between the unit circle $|x|=1$ and $\mathfrak{X}$, if $\frac{\mu_{1}}{\lambda_{1}}>1$, and, if $\frac{\mu_{1}}{\lambda_{1}}<1$, then $R_{x}$ is simply the unit disk $\mathcal{D}_{x}(0,1)$. Last

From Proposition 3.9 (Lemma 2.3. in [3]) we have:

$$
\begin{aligned}
& X_{0}\left(Y_{0}(x)\right)=\left\{\begin{array}{cc}
x & \text { if }|x| \leq \sqrt{\frac{\mu_{1}}{\lambda_{1}}} \\
\frac{\mu_{1}}{\lambda_{1} x} & \text { if }|x|>\sqrt{\frac{u_{1}}{\lambda_{1}}}
\end{array}\right. \\
& Y_{0}\left(X_{0}(y)\right)=\left\{\begin{array}{cc}
y & \text { if }|y| \leq \sqrt{\frac{\mu_{2}}{\lambda_{2}}} \\
\frac{\mu_{2}}{\lambda_{2} y} & \text { if }|y|>\sqrt{\frac{u_{2}}{\lambda_{2}}}
\end{array} .\right.
\end{aligned}
$$

Using the above lemma along with the regions we have just described, we see that, if $\frac{\mu_{2}}{\lambda_{2}}>1$ and $\frac{\mu_{1}}{\lambda_{1}}>1$, then there is a bijection between $R_{y}$ and $R_{x}$, via $Y_{0}(x)$ and $X_{0}(y)$.

If $\frac{\mu_{2}}{\lambda_{2}}<1$ and $\frac{\mu_{1}}{\lambda_{1}}>1$ then there is a bijection between $G_{y}$ and the domain between the unit circle $|x|=1$ and $\mathfrak{X}$.

Note: The case where both $\frac{\mu_{2}}{\lambda_{2}}<1$ and $\frac{\mu_{1}}{\lambda_{1}}<1$ is rejected, otherwise, as soon as both queues become busy, the queue lengths will remain unbounded and the system will blow up. Therefore, we will assume that $\mu_{1}>\lambda_{1}$.

From the details and results we have given insofar in this section, we can conclude Lemma 3.1 in [3], which states that all the couples $(x, y)$ which are 
solutions to $h(x, y)=0$ with $|x| \leq 1$ and $|y| \leq 1$ have the form $\left(x, Y_{0}(x)\right)$ or $\left(X_{0}(y), y\right)$ where $x \in R_{x}$ and $y \in R_{y}$.

\section{Analytic Continuation of $\pi_{1}(x)$ :}

We are now in a good position to demonstrate the analytic continuation of $\pi_{1}(x)$. Symmetric methods and results can be made for $\pi_{2}(y)$, so we will only present detailed analysis for $\pi_{1}(x)$. The objective is to analytically continue $\pi_{1}(x)$ past the unit circle in $\mathbb{C}_{x}$, but we can do better. We can show that $\pi_{1}(x)$ can be continued as a meromorphic function to the whole complex plane cut along the real axis from $x_{3}$ to $x_{4}$ whose poles, if the exist, are the roots of $h_{1}\left(x, Y_{0}(x)\right)$ and these roots $\in \overline{\mathcal{D}}_{x}(0,1)^{c}$. The demonstration of the analytic continuation is as follows:

For $|x|<1$ and $|y|<1$ we have,

$$
h_{1}(x, y) \pi_{1}(x)+h_{2}(x, y) \pi_{2}(y)+h_{0}(x, y) \pi_{0,0}=0 .
$$

It is clear that $\pi_{1}(x)$ is analytic for $|x|<1$, so we want to continue the function to the region $\overline{\mathcal{D}}_{x}(0,1)^{c}$, that is, everything outside the unit disk and unit circle. Again, we assume that $\frac{\mu_{1}}{\lambda_{1}}>1$ and we consider the first of two cases.

1. If $\frac{\mu_{2}}{\lambda_{2}}<1$ :

From our preliminary work, we know that $Y_{0}(x)$ is analytic in the region $\overline{\mathcal{D}}_{x}(0,1)^{c}$ cut along $\left[x_{3}, x_{4}\right]$ and $\left|Y_{0}(x)\right| \leq \sqrt{\frac{\mu_{2}}{\lambda_{2}}}<1$ for all $x$, so that, when using 
the equation:

$$
h_{1}\left(x, Y_{0}(x)\right) \pi_{1}(x)+h_{2}\left(x, Y_{0}(x)\right) \pi_{2}\left(Y_{0}(x)\right)+h_{0}\left(x, Y_{0}(x)\right) \pi_{0,0}=0
$$

we can easily see that,

$$
h_{2}\left(x, Y_{0}(x)\right) \cdot \pi_{2}\left(Y_{0}(x)\right) \text { and } h_{0}\left(x, Y_{0}(x)\right) \cdot \pi_{0,0}
$$

are all analytic in $\overline{\mathcal{D}}_{x}(0,1)^{c}$, cut along $\left[x_{3}, x_{4}\right]$. Note that the product of two analytic functions gives an analytic function [12]. At this point, we can use the Identity Theorem to conclude that the product $h_{1}\left(x, Y_{0}(x)\right) \cdot \pi_{1}(x)$ is analytic in $\overline{\mathcal{D}}_{x}(0,1)^{c}$.

Suppose we want to further isolate $\pi_{1}(x)$ and use the Identity Theorem to solely continue $\pi_{1}(x)$. First we assume that $h_{1}\left(x, Y_{0}(x)\right) \neq 0$, for $|x|<1$, so that we have:

$\pi_{1}(x)=\frac{h_{2}\left(x, Y_{0}(x)\right) \pi_{2}\left(Y_{0}(x)\right)+h_{0}\left(x, Y_{0}(x)\right) \pi_{0,0}}{h_{1}\left(x, Y_{0}(x)\right)}$.

If we assume that $h_{1}\left(x, Y_{0}(x)\right) \neq 0$ for all $x$, then we can easily use the Identity Theorem and analytically continue $\pi_{1}(x)$ to its nearest singularity, which would be the branch point $x_{3}$. If we speak of meromorphic continuation, then we include the possibility that $h_{1}\left(x, Y_{0}(x)\right)$ has roots inside $\left(1, x_{3}\right)$ and then we can say that $\pi_{1}(x)$ has been meromorphically continued to the region $\overline{\mathcal{D}}_{x}(0,1)^{c}$ cut along $\left[x_{3}, x_{4}\right]$ with 
potential poles at the points where both $h_{1}\left(x, Y_{0}(x)\right)=0$ and $h\left(x, Y_{0}(x)\right)=0$.

If $\frac{\mu_{2}}{\lambda_{2}}>1$ :

This situation is a bit trickier, since, as previously shown, the region that $Y_{0}(x)$ maps into contains the unit disk $\mathcal{D}_{y}(0,1)$. In first case above we knew that $\left|Y_{0}(x)\right|<1$ for every $x \in \mathbb{C}_{x}$ and this made analytic continuation almost trivial. In this case, $Y_{0}(x)>1$ for some $x \in \mathbb{C}_{x}$. We begin by letting $\mathfrak{S}$ denote the region inside the curve $\mathfrak{X}^{\prime}$, that is, the region in $\mathbb{C}_{x}$ where $x \in \mathcal{D}_{z}(0,1)^{c}$ and $\left|Y_{0}(x)\right| \geq 1$. We see that the branch points $x_{3}$ and $x_{4}$ are interior points of $\mathfrak{S}$ and in the region $\overline{\mathcal{D}}_{z}(0,1)^{c} \backslash \mathfrak{S},\left|Y_{0}(x)\right| \leq 1$, so that the product $\pi_{1}(x) \cdot h_{1}\left(x, Y_{0}(x)\right)$ is analytic in the region $\overline{\mathcal{D}}_{z}(0,1)^{c} \backslash \mathfrak{S}$.

Now we want the analytic continuation of $\pi_{1}(x)$ to $\mathfrak{S} \backslash\left[x_{3}, x_{4}\right]$. We first use the fundamental form and the fact that $\pi_{1}(x)$ is analytic in the unit disk to continue $\pi_{2}\left(Y_{0}(x)\right)$ to the unit circle $|x|=1$ cut along $\left[x_{1}, x_{2}\right]$ in the exact same fashion as we did above.

Now it is clear that $Y_{0}(x)=Y_{0}\left(\frac{\mu_{1}}{\lambda_{1} x}\right)$ and it can be shown that $\left[x_{3}, x_{4}\right]=\frac{\mu_{1}}{\lambda_{1}\left[x_{1}, x_{2}\right]}$.

Therefore, $\pi_{2}\left(Y_{0}(x)\right)$ is continued as a meromorphic function to $\mathfrak{S} \backslash\left[x_{3}, x_{4}\right]$ and again, by the fundamental form, we get the conclusion that the product $\pi_{1}(x)$. $h_{1}\left(x, Y_{0}(x)\right)$ is analytic in the whole complex plane cut along $\left[x_{3}, x_{4}\right]$ and $\pi_{1}(x)$ is meromorphic in the whole complex plane cut along $\left[x_{3}, x_{4}\right]$ whose poles are the roots of both $h_{1}\left(x, Y_{0}(x)\right)$ and $h\left(x, Y_{0}(x)\right)$ in the region $\overline{\mathcal{D}}_{z}(0,1)^{c} \backslash\left[x_{3}, x_{4}\right]$. Identical 
results can be drawn for $\pi_{2}(y)$.

What conclusions can we draw from this analysis? The reason why this particular model provided an elegant method to analytically continue $\pi_{1}(x)$ is due to the fact that the contour $\partial D_{y} \equiv|y|=\sqrt{\frac{\mu_{2}}{\lambda_{2}}}$ is a circle. We know by the Maximum Modulus Principle that the value of $\left|Y_{0}(x)\right|$ reaches its maximum on the boundary of $\left[x_{1}, x_{2}\right]$. Therefore, when $|y|=\sqrt{\frac{\mu_{2}}{\lambda_{2}}}<1$, we know that $\left|Y_{0}(x)\right| \leq \sqrt{\frac{\mu_{2}}{\lambda_{2}}}<1$ is true for every $x \in \mathbb{C}_{x}$. Then analytic continuation is trivial. When $\partial D_{y} \equiv|y|=\sqrt{\frac{\mu_{2}}{\lambda_{2}}}>1$, we had to do a little more work. But the idea stayed the same: we set up our regions, took complements, which were easily described because the contours were circles, used automorphisms, and we were back in regions that we could use. Notice that we had to continue $\pi_{2}(y)$ first in the second case in order to continue $\pi_{1}(x)$ and we were able to achieve that by using the results of the first case.

Also note that we were able to factor out and cancel an $x$ when simplifying the expression for $Y_{ \pm}(x)$. In fact, we know on the branch cuts that $Y_{0}(x)$ and $Y_{1}(x)$ are complex conjugates and so their moduli are equal. Indeed, for any $x$ on a branch cut, we have:

$$
\begin{aligned}
& Y_{ \pm}(x)=\frac{-b(x) \pm i \sqrt{4\left(a(x) c(x)-b(x)^{2}\right.}}{2 a(x)}, \quad \text { which gives, } \\
& \left|Y_{ \pm}(x)\right|=\sqrt{\frac{c(x)}{a(x)}}
\end{aligned}
$$

We immediately see that $\left|Y_{ \pm}(x)\right|$ will be a constant if $a(x)$ and $c(x)$ have one term each and the degree of $a(x)$ and $c(x)$ are equal. Furthermore, by rationalizing the denominator, we can easily show that $Y_{0}(x)=\frac{c(x)}{a(x) Y_{1}(x)}$. When $\frac{c(x)}{a(x)}$ is a constant, 
then we get a clean expression for $Y_{1}(x)$ and a nice conformal mapping, which is simply an inversion map, between the two regions produced by $Y_{0}(x)$ and $X_{0}(y)$.

Conclusion: If we have a stable, light-tailed, non-singular random walk and if $a(x)$ and $b(x)$ each have one term with the same degree, then we can use the method described in this section to analytically continue both generating functions $\pi_{1}(x)$ and $\pi_{2}(y)$ past the unit disk, in their respective planes, and meromorphically continue $\pi_{1}(x)$ and $\pi_{2}(y)$ to the whole complex cut plane.

The next section explores the situation where the contour $\partial D_{x}$ and $\partial D_{y}$ are not nice circles. 


\subsection{Wireless Three-Hop Networks Model}

This is a summary of the method employed for analytically continuing the generating function $\Pi_{1}(x)$ for the wireless three-hop networks model, which is a discretetime two-dimensional Markov chain, in the paper, Wireless three-hop networks with stealing II: exact solutions through boundary value problems [5].

Using familiar notation, the transition probabilities are:

$$
\begin{array}{ll}
p_{1,0}=\frac{1-p}{3}, & p_{0,-1}=\frac{1+p}{3}, \quad p_{-1,1}=3, \\
p_{1,0}^{(1)}=\frac{1}{2}, & p_{-1,1}^{(1)}=\frac{1}{2} \\
p_{1,0}^{(2)}=\frac{1-p}{2}, & p_{0,-1}^{(2)}=\frac{1+p}{2}, \\
p_{1,0}^{(0)}=1 . &
\end{array}
$$

Again, we then know that:

1. $\Pi(x, y)$ is analytic in the open domain $\left\{(x, y) \in \mathbb{C}^{2}:|x|<1,|y|<1\right\}$,

2. $\Pi(x, y)$ is continuous in the closed domain $\left\{(x, y) \in \mathbb{C}^{2}:|x| \leq 1,|y| \leq 1\right\}$.

Moreover, we have the fundamental form:

$$
H(x, y) \Pi(x, y)=H_{1}(x, y) \Pi_{1}(x)+H_{2}(x, y) \Pi_{2}(y)+H_{0}(x, y) \pi_{0,0},
$$


where:

$H(x, y)(x, y)=6 x y-2(1-p) x^{2} y-2 y^{2}-2(1+p) x$,

$H_{1}(x, y)(x, y)=(1+2 p) x^{2} y+y^{2}-2(1+p) x$,

$H_{2}(x, y)(x, y)=(1-p) x^{2} y-2 y^{2}+(1+p) x$,

$H_{0}(x, y)(x, y)=(2+p) x^{2} y-y^{2}-(1+p) x$.

Based on Theorem 2.2, we can easily show that the random walk is stable if and only if $p \in(0,1]$ and we will assume that the stability condition for this random walk holds.

For fixed $y$, the equation $H(x, y)=0$ has the two roots in the variable $x$ :

$$
X_{ \pm}(y)=\frac{-(1+p-3 y) \pm \sqrt{\tilde{\delta}_{2}(y)}}{2(1-p) y}
$$

where the discriminant, $\tilde{\delta}_{2}(y)$, is defined as:

$$
\tilde{\delta}_{2}(y)=(1+p-3 y)^{2}-4(1-p) y^{3},
$$

and, for fixed $x$, the two roots in the variable $y$ are:

$$
Y_{ \pm}(x)=\frac{(3-(1-p) x) x \pm \sqrt{\delta_{1}(x)}}{2}
$$


where the discriminant, $\delta_{1}(x)$, is:

$$
\delta_{1}(x)=(3-(1-p) x)^{2} x^{2}-4(1+p) x
$$

We quickly notice that $X_{ \pm}(y)$ has a singularity when its denominator 2(1 $p) y=0$. To classify the singularity, we note that:

$\lim _{y \rightarrow 0} X_{+}(y)=\frac{-(1+p)+\sqrt{(1+p)^{2}}}{2(1-p) y}=0$.

Since the point $\alpha \in \mathbb{C}$ is a removable singularity of the complex function $f(z)$ if and only if $\lim _{z \rightarrow \alpha} f(z)$ exists [12], we conclude that the point 0 is a removable singularity.

On the other hand,

$\lim _{y \rightarrow 0^{+}} X_{-}(y)=\frac{-(1+p)-\sqrt{(1+p)^{2}}}{2(1-p) y}=-\frac{1}{y}=-\infty$.

Therefore, the point 0 is a pole for $X_{-}(y)$.

We can use Theorem 3.1 and Corollary 3.2 to show that $X_{ \pm}(y)$ has 3 real branch points such that $0<y_{1}<y_{2}<1<y_{3}$ with $y_{4}=\infty$. Similarly, $Y_{ \pm}(x)$ has 4 real branch points such that $0=x_{1}<x_{2}<1<x_{3}<x_{4}<\infty$. However, if we further investigate the properties of $\tilde{\delta}(y)$ and $\delta(x)$, then we can get an upper bound for $y_{1}$ 
that is smaller $y_{2}$ and an upper bound for $x_{3}$ that is smaller than $x_{4}$, respectively.

Indeed, because $\tilde{\delta}(y)$ is a polynomial of degree three, and,

$\tilde{\delta}(0)>0 \quad \tilde{\delta}\left(\frac{1}{3}(1+p)\right)<0 \quad \tilde{\delta}(1)=p^{2}>0$,

the 3 real roots are positioned on the real line as:

$0<y_{1}<\frac{1}{3}(1+p)<y_{2}<1<y_{3}$

Again, note that $\tilde{\delta}(y)>0$ when $y \in\left(-\infty, y_{1}\right) \cup\left(y_{2}, y_{3}\right)$.

Moreover, when $y \in\left(y_{1}, y_{2}\right) \cup\left(y_{3}, \infty\right), \tilde{\delta}(y)<0$ and $X_{ \pm}(y)$ are complex conjugate roots and we know that the image of the segment $\left[y_{1}, y_{2}\right]$ is a closed contour $\partial D_{x}$ that is symmetric with respect to the horizontal axis in the $\mathbb{C}_{x}$ plane.

For $Y_{ \pm}(x)$, the only singularities that exist are its branch points.

In this case, we get the 4 real roots, positioned on the real line as:

$0=x_{1}<x_{2}<1<x_{3}<3 \frac{1}{(1-p)}<x_{4}$.

Again, $\delta(x)>0$ when $x \in\left(-\infty, x_{1}\right) \cup\left(x_{2}, x_{3}\right) \cup\left(x_{4}, \infty\right)$.

Furthermore, when $x \in\left(x_{1}, x_{2}\right) \cup\left(x_{3}, x_{4}\right), \delta(x)<0, Y_{ \pm}(x)$ are complex conjugate roots, and the image of the segment $\left[x_{1}, x_{2}\right]$ is a closed contour $\partial D_{y}$ that is symmetric to the horizontal axis in the $\mathbb{C}_{y}$ plane. Moreover, according to Theorem 
3.3 and 3.4 we have the following:

The function

$$
X_{0}(y)= \begin{cases}X_{+}(y), & \text { if } y \in\left\{z: \Re(z) \leq y_{2}, \Im\left(\tilde{\delta}\left(z^{+}\right)\right)<0\right\} \cup\left(-\infty, y_{1}\right) \\ X_{-}(y), & \text { otherwise }\end{cases}
$$

is analytic in $\mathbb{C} \backslash\left[y_{1}, y_{2}\right] \cup\left[y_{3}, \infty\right]$.

The function

$$
X_{1}(y)= \begin{cases}X_{-}(y), & \text { if } y \in\left\{z: \Re(z) \leq y_{2}, \Im\left(\tilde{\delta}\left(z^{+}\right)\right)<0\right\} \cup\left(-\infty, y_{1}\right) \\ X_{+}(y), & \text { otherwise }\end{cases}
$$

is meromorphic in $\mathbb{C} \backslash\left[y_{1}, y_{2}\right] \cup\left[y_{3}, \infty\right]$ with a simple pole at 0

The function

$$
Y_{0}(x)= \begin{cases}Y_{+}(x), & \text { if } x \in\left\{z: \Re(z) \leq x_{2}, \Im\left(\delta\left(z^{+}\right)\right)<0\right\} \cup\left(-\infty, x_{1}\right) \\ Y_{+}(x), & \text { if } x \in\left\{z: \Re(z) \geq x_{3}, \Im\left(\delta\left(z^{+}\right)\right)>0\right\} \cup\left(x_{4}, \infty\right) \\ Y_{-}(x), & \text { otherwise }\end{cases}
$$

is analytic in $\mathbb{C} \backslash\left[x_{1}, x_{2}\right] \cup\left[x_{3}, x_{4}\right]$.

The function

$$
Y_{1}(x)= \begin{cases}Y_{-}(x), & \text { if } x \in\left\{z: \Re(z) \leq x_{2}, \Im\left(\delta\left(z^{+}\right)\right)<0\right\} \cup\left(-\infty, x_{1}\right) \\ Y_{-}(x), & \text { if } x \in\left\{z: \Re(z) \geq x_{3}, \Im\left(\delta\left(z^{+}\right)\right)>0\right\} \cup\left(x_{4}, \infty\right) \\ Y_{+}(x), & \text { otherwise }\end{cases}
$$

is analytic in $\mathbb{C} \backslash\left[x_{1}, x_{2}\right] \cup\left[x_{3}, x_{4}\right]$.

We will now try and locate the contours $\partial D_{x}$ and $\partial D_{y}$, produced by $Y_{0}(x)$ and 
$X_{0}(y)$ along their branch cuts in the unit disk, respectively.

The closed contour $\partial D_{x}$ outlines the bounded domain $D_{x}$ symmetric with respect to the horizontal axis in the $\mathbb{C}_{x}$ plane and $\partial D_{x}$ can be parametrized by

$\partial D_{x}=\left\{\left(\frac{-(1+p-3 y) \pm i \sqrt{-\Delta_{2}(y)}}{2(1-p) y}\right), y \in\left[y_{1}, y_{2}\right]\right\}$

Now, $(1+p-3 y) \geq 0$ for $y \in\left[y_{1}, \frac{1}{3}(1+p)\right]$, which implies that the real part of the points $x$ on $\partial D_{x}$ is non positive. Conversely, $(1+p-3 y) \leq 0$ for $y \in\left[\frac{1}{3}(1+p), y_{2}\right]$, which implies that the real part of the points $x$ on $\partial D_{x}$ is positive and the image of the interval $\left[y_{2}, 1\right]$, by either $X_{0}(y)$ or $X_{1}(y)$ are intervals on $\mathbb{R}_{+}$.

Also, since $(1+p-3 y)$ is a decreasing function on the interval $\left[y_{1}, \frac{1}{3}(1+p)\right]$, the absolute value of the real part of the points $x$ on $\partial D_{x}$ is decreasing on the interval $\left[y_{1}, \frac{1}{3}(1+p)\right]$. Therefore, $X_{0}\left(y_{1}\right) \leq 0$ is the extreme left point of $\partial D_{x}$. It can be shown that $X_{0}\left(y_{1}\right)=-\sqrt{\frac{y_{1}}{(1-p)}}$.

On account of $H(1,1)=0$, we deduce that either $X_{0}(1)=1$ or $X_{1}(1)=1$, which implies that 1 belongs to the image of either $X_{0}\left[y_{2}, 1\right]$ or $X_{1}\left[y_{2}, 1\right]$. Note that this image is a closed interval containing $x_{2}$. Now take an $x$ which belongs to this closed interval. Then $Y_{0}(x)$ and $Y_{1}(x)$ are defined and we can conclude that $X_{0}\left(y_{2}\right)=X_{1}\left(y_{2}\right) \geq x_{2}$. In fact, $X_{0}\left(y_{2}\right)$ is at the extreme right of the contour $\partial D_{x}$. Conceptually, this indicates that the contour $\partial D_{x}$ encompasses the interval $\left[x_{1}, x_{2}\right]$.

Similarly, the closed contour $\partial D_{y}$ outlines a bounded domain $D_{y}$ symmetric with respect to the horizontal axis in the $\mathbb{C}_{y}$ plane and we can parametrize $\partial D_{y}$ 
in the same fashion as we did for $\partial D_{x}$. In this case though, the extreme left point is $Y_{0}\left(x_{1}\right)=Y_{0}(0)=0$. Furthermore, since

$\delta\left(\frac{1}{1+p}\right)=\frac{(2+4 p)^{2}}{(1+p)^{4}}-4<0$,

$x_{2}>\frac{1}{1+p}$ and the right extreme point of the contour $\partial D_{y}$ is $\sqrt{x_{2}(1+p)}$.

Remark: We have made some claims concerning the right extreme points of the contours that we should justify. If we take an $x \in\left(x_{1}=0, x_{2}\right)$, then $\delta(x)<0$, and we have:

$$
Y_{ \pm}(x)=\frac{(3-(1-p) x) x \pm i \sqrt{-\delta(x)}}{2}
$$

so that $\left|Y_{ \pm}(x)\right|=\sqrt{\Re\left(Y_{ \pm}(x)\right)^{2}+\Im\left(Y_{ \pm}(x)\right)^{2}}<\sqrt{(1+p) x}$. We immediately see that the modulus of $\left|Y_{ \pm}(x)\right|$ is dependent on $x$ and as $x$ increases, $\sqrt{(1+p) x}$ increases. On the slit, not including the branch points, the kernel function, for a given $x$, has two complex conjugate roots. Hence, their magnitudes must be equal, and that magnitude is less than $\sqrt{(1+p) x}$, which is a function of $x$. Again, the fact that they are complex conjugates gives us a contour that symmetric on the horizontal axis. At the branch point $x_{2}, Y_{0}\left(x_{2}\right)=Y_{1}\left(x_{2}\right)$ and we get, at $x_{2}$ :

$$
Y_{ \pm}\left(x_{2}\right)=\frac{\left(3-(1-p) x_{2}\right) x_{2}}{2}
$$

From the above, we can show that the extreme right point of the contour $\partial D_{y}$ is 
$\sqrt{(1+p) x_{2}}$. An identical argument can be put forth for the contour $\partial D_{y}$.

Finally, by Theorem 3.8, since $\Delta=\frac{(1+p)(1-p)}{27}>0$ if we let $p \in(0,1)$ and we therefore know that $\left[x_{1}, x_{2}\right] \subset D_{x}$ and $\left[y_{1}, y_{2}\right] \subset D_{y}$. Furthermore, by Proposition 3.9 we have $X_{0}\left(\partial D_{y}\right) \subset\left[x_{1}, x_{2}\right]$ and $Y_{0}\left(\partial D_{x}\right) \subset\left[y_{1}, y_{2}\right]$.

We are now in a good position to properly demonstrate the analytic continuation of $\Pi_{1}(x)$. The reason why we were thorough with our presentation of the contours $\partial D_{x}$ and $\partial D_{y}$ is because we will be analytically continuing the generating functions to the regions bounded by these contours. The performance of the analytic continuation of $\Pi_{1}(x)$ will play out a bit differently than it did in the last section.

\section{Analytic Continuation of $\Pi_{1}(x)$}

We would like to show that $\Pi_{1}(x)$ is meromorphic in $D_{x} \backslash\left[x_{1}, x_{2}\right]$ with a simple pole $x^{*}$ when $x^{*}=X_{0}\left(y^{*}\right)$; otherwise, it is analytic in $D_{x} \backslash\left[x_{1}, x_{2}\right]$.

To prove the preceding claim, we first recall that $\Pi_{1}(x)$ is analytic for all $x \in$ $\mathcal{D}_{x}(0,1)$. Let $\mathfrak{N}(\alpha)$ denote an open neighborhood of the point $\alpha$. Then for $y \in$ $\mathfrak{N}\left(0^{+}\right) \subset D_{y} \backslash\left[y_{1}, y_{2}\right], X_{0}(y) \in \mathfrak{N}(0) \subset \mathcal{D}_{x}(0,1)$. Therefore, for $y \in \mathfrak{N}(0)$, all of the components in the fundamental form (2.2) are well defined and finite, and we get:

$$
H_{1}\left(X_{0}(y), y\right) \Pi_{1}\left(X_{0}(y)\right)+H_{2}\left(X_{0}(y), y\right) \Pi_{2}(y)+H_{0}\left(X_{0}(y), y\right) \pi_{0,0}=0
$$

Naturally, we are inclined to take the next step, by solving for $\Pi_{1}\left(X_{0}(y)\right)$ in the above equation. However, by doing so, we have to divide the left hand side by $H_{1}\left(X_{0}(y), y\right)$, and this new denominator can produce a potential singularity at some point $y$ if, for some $x, H(x, y)=0$ and $H_{1}(x, y)=0$. In the paper, the 
authors used the resultant of the curves $H(x, y)$ and $H_{1}(x, y)$ to determine the points of intersection. For the purpose of this proof, we shall let $x^{*}=X_{0}\left(y^{*}\right)$ be the point such that $H\left(x^{*}, y\right)=0$ and $H_{1}\left(x^{*}, y\right)=0$.

If $x^{*} \neq X_{0}\left(y^{*}\right)$, then, for $y \in \mathfrak{N}(0)$,

$$
\Pi_{1}\left(X_{0}(y)\right)=\frac{H_{2}\left(X_{0}(y), y\right) \Pi_{2}(y)+H_{0}\left(X_{0}(y), y\right) \pi_{0,0}}{H_{1}\left(X_{0}(y), y\right)}
$$

Let us now look at the region $\left\{\mathcal{D}_{y}(0,1) \cap D_{y} \backslash\left[y_{1}, y_{2}\right]\right\} \subset \mathcal{D}_{y}(0,1)$. When we take a $y \in \mathcal{D}_{y}(0,1) \cap D_{y} \backslash\left[y_{1}, y_{2}\right]$, then obviously $y \in \mathcal{D}_{y}(0,1)$ and therefore $\Pi_{2}(y)<$ $\infty$. Moreover, each component in (4.2) is analytic in $\mathcal{D}_{y}(0,1) \cap D_{y} \backslash\left[y_{1}, y_{2}\right]$ and $\left|X_{0}(y)\right|<1$. This demonstrates, via (4.2), that $\Pi(x)$ can be analytically continued in the domain $X_{0}\left(\left(D_{y}(0,1)\right) \cap D_{y} \backslash\left[y_{1}, y_{2}\right]\right)$. More concretely, we are interested in the interval $\left(y_{2}, 1\right)$ since, not only is $X_{0}(y)$ analytic on this line segment, but $X_{0}\left(y_{2}, 1\right)=\left(1, X_{0}\left(y_{2}\right)\right)$ and this extends beyond the original domain of analyticity for $\Pi_{1}(x)$. If $X_{0}(y)$ is analytic at the branch point $y_{2}$, then we have shown, assuming $x^{*} \neq X_{0}\left(y^{*}\right)$, that $\Pi_{1}(x)$ can be analytically continued to the whole of $D_{x} \backslash\left[x_{1}, x_{2}\right]$.

On the other hand, $X_{0}(y)$ may not be analytic at the branch point $y_{2}$ and then we can not immediately conclude that $\Pi_{1}(x)$ is analytic at $X_{0}\left(y_{2}\right)$. To rectify this, we note that $\Pi_{1}(x)$ is analytic in $\mathcal{D}_{x}(0,1)$ so we know that $\Pi_{1}(x)$ has a convergent power series expansion around $x=0$ and this power series has positive coefficients. Furthermore, for $y_{2} \in \mathbb{D}_{y}(0,1), \Pi_{1}\left(x_{2}\right)<\infty$ and $\Pi_{1}\left(X_{0}(y)\right)$ is continuous at the point $y_{2}$ and so $\Pi_{1}\left(X_{0}\left(y_{2}\right)\right)<\infty$. Since, the coefficients of the power series are real numbers, we can restrict both the power series and $\Pi_{1}(x)$ to the real numbers. 
We also know from Theorem 4.3 that the radius of convergence $R$ for the power series is a singular point of $\Pi_{1}(x)$. If the real point $R$ is a singularity of $\Pi_{1}(x)$ then $X_{0}\left(y_{2}\right) \leq R$, since otherwise $\Pi_{1}(x)$ would not be analytic in $\left(1, X_{0}\left(y_{2}\right)\right)$. We also know that $\Pi_{1}\left(X_{0}\left(y_{2}\right)\right)<\infty$, so $\left(X_{0}\left(y_{2}\right)\right) \neq R$. Therefore, $X_{0}\left(y_{2}<R\right.$ and the power series must be convergent at the point $X_{0}\left(y_{2}\right.$. This again shows that $\Pi_{1}(x)$ can be analytically continued to $\left(1, X_{0}\left(y_{2}\right)\right)$ and from there we can continue to the region $D_{x} \backslash\left[x_{1}, x_{2}\right]$.

It should be noted again that the authors found the values and properties of $x^{*}$ and $y^{*}$ using the resultant of $H(x, y)$ and $H_{1}(x, y)$. For what follows, we will simply assume the values and properties of $x^{*}$ and $y^{*}$ without proof. In analyzing $x^{*}$ and $y^{*}$, the authors found that the equation $H_{2}\left(x, Y_{0}(x)\right)=0$ has no solution in both $\mathcal{D}_{x}(0,1)$ and the region $D_{x}$. Moreover, the point $x^{*}$ is a removable singularity for the function $H_{1}\left(x, Y_{0}(x)\right) \Pi_{1}(x)$.

Now we assume that $x^{*}=X_{0}\left(y^{*}\right)$, which implies that, $\Pi_{1}(x)$ may have a pole at $x^{*} \in D_{x}$. We know that $H_{1}\left(X_{0}(y), y\right) \Pi_{1}\left(X_{0}(y)\right)$ is analytic at $y^{*}$. Moreover, we also know that for $x \in \mathfrak{N}(0) \subset \mathcal{D}_{x}(0,1), Y_{0}(x) \in \mathfrak{N}(0) \subset \mathcal{D}_{y}(0,1)$. Then, for $x \in \mathfrak{N}(0)$, we have:

$$
\Pi_{2}\left(Y_{0}(x)\right)=\frac{H_{1}\left(x, Y_{0}(x)\right) \Pi_{1}(x)+H_{0}\left(x, Y_{0}(x)\right) \pi_{0,0}}{H_{2}\left(x, Y_{0}(x)\right)} .
$$

As we did for $\Pi_{1}(x)$, the above equation permits us to analytically continue $\Pi_{2}(y)$ into the region $Y_{0}\left(\mathcal{D}_{x}(0,1) \cap\left[x_{1}, x_{2}\right]\right)$. Since $Y_{0}\left(\left(x_{2}, 1\right)\right)=\left(1, Y_{0}\left(x_{2}\right)\right), \Pi_{2}(y)$ can be analytically continued along the segment $\left(1, Y_{0}\left(x_{2}\right)\right)$ and, since $\Pi_{2}\left(Y_{0}\left(x_{2}\right)<\right.$ $\infty$, we can use the same arguments as above and analytically continue $\Pi_{2}(y)$ to 
the domain $D_{y} \backslash\left[y_{1}, y_{2}\right]$. Knowing this allows us to turn back to the fundamental form and see that $\Pi_{1}(x)$ is meromorphic in $D_{x} \backslash\left[x_{1}, x_{2}\right]$

Conclusion: This example was harder than the first example in the last section. One of the main differences was that the contours were not circles. However, we were still able to construct the contours and determine one of their key features: the extreme left and right points. These points are real and at least one of them sits outside the unit circle. We were then able to analytically continue the generating functions, pass the unit disk, and extend them into the regions bounded by the contours. What enable us to do that was, we used a stable non-singular random walk with light-tailed stationary distributions, and we showed that:

1. $Y_{0}\left(\left(x_{2}, 1\right)\right)=\left(1, Y_{0}\left(x_{2}\right)\right)$ with $Y_{0}\left(x_{2}\right)>1$ and $X_{0}\left(\left(y_{2}, 1\right)\right)=\left(1, X_{0}\left(y_{2}\right)\right)$ with $X_{0}\left(y_{2}\right)>1$,

2. Irrespective of whether $Y_{0}(x)$ is analytic at $x_{2}$ and $X_{0}(y)$ is analytic at $y_{2}$, $\pi_{2}\left(Y_{0}\left(x_{2}\right)\right)<\infty$ and $\pi_{1}\left(X_{0}\left(y_{2}\right)\right)<\infty$.

3. $h_{2}\left(x, Y_{0}(x)\right.$ does not have a root in $\left(1, X_{0}\left(y_{2}\right)\right)$ and $h_{1}\left(X_{0}(y), y\right)$ does not have a root in $\left(1, Y_{0}\left(x_{2}\right)\right)$

We were then able to use the fundamental form and first extend the generating functions along the line segment $\left(1, X_{0}\left(y_{2}\right)\right)$ and $\left(1, Y_{0}\left(x_{2}\right)\right)$, respectively, and continuing to the domains $D_{x} \backslash\left[x_{1}, x_{2}\right]$ and $D_{y} \backslash\left[y_{1}, y_{2}\right]$. 


\section{Chapter 5}

\section{Conclusion and Future Work}

\subsection{Conclusion}

The assumption throughout this thesis was that both the stationary distributions and the generating functions of the random walk in the quarter plane were unknown. Since the generating functions encode the stationary distributions and we have a functional equation linking the generating functions, we naturally chose to work with the generating functions to gather information about the stationary distributions. We provided examples of methods used to either solve these generating functions, whether it be in a closed form expression or an integral representation, or to derive asymptotic properties about the generating functions. Most methods, in general, require that we make $\pi_{1}(x)$ and $\pi_{2}(y)$ analytic in a larger domain than the unit disk. Lemma 2.2.1 in [2] shows that if $\mathbf{M} \neq 0$ and $h(x, y)$ is irreducible, then there exists an $\epsilon>0$ such that $\pi_{1}(x)$ and $\pi_{2}(y)$ can 
be analytically continued up to the closed disk $\overline{\mathcal{D}}(0,1+\epsilon)$, in their respective complex plane, and that the fundamental form is satisfied in $\mathcal{B} \cap \overline{\mathcal{D}}(0,1+\epsilon)^{2}$. However, the proof for this assertion is intricate and requires a strong knowledge of various complex topics swimming in the sea of mathematics. Our goal was to use minimal results derived from not so intricate mathematics and some complex analysis to demonstrate the same result as Lemma 2.2.1: for a light-tailed nonsingular random walk, we can analytically continued $\pi_{1}(x)$ and $\pi_{2}(y)$ to at least the domain $D_{x} \backslash\left[x_{1}, x_{2}\right]$ and $D_{y} \backslash\left[y_{1}, y_{2}\right]$, respectively. We achieved this by using the fundamental form and having a good understanding of the branches $Y_{0}(x)$ and $X_{0}(y)$ and the contours produced by $Y_{0}(x)$ and $X_{0}(y)$ along their branch cuts $\left[x_{1}, x_{2}\right]$ and $\left[y_{1}, y_{2}\right]$, respectively.

\section{$5.2 \quad$ Future Work}

The chief reason why we wanted to explore the idea of using a simpler method in analytically continuing the generating functions is so we can potentially extend the results to random walks that are modeled in higher dimensions. Many of the theorems employed for two-dimensional random walks fail to hold in higher dimensions. I would really enjoy further researching how far into the complex plane we can continue $\pi_{1}(x)$ and $\pi_{2}(y)$ using the same train of thought as was presented in this thesis and also examining whether we can extend these methods consistently for $n$ th-dimensional random walks. 


\section{Bibliography}

[1] Dai, J.G. and Miyazawa, Masakiyo, (2011) Stationary distribution of a twodimensional SRBM: geometric views and boundary measures, submitted.

[2] Fayolle, Guy, Iasnogorodski, Roudolf, and Malyshev, Vadim, (1999) Random Walk in the Quarter Plane, Springer, New York.

[3] Fayolle, Guy and Iasnogorodski, Roudolf, (1979) Two coupled processors: the reduction to a Riemann-Hilbert problem, Z. Wahrscheinlichkeitsth, 47, 325351.

[4] Fleming, Wendell, (1977) Functions of Several Variables, springer-Verlag, New York.

[5] Guillemin, Fabrice, Knessl, Charles, and Leeuwaarden, Johan S.H. van, (2012) Wireless three-hop networks with stealing II: exact solutions through boundary value problems Queueing Systems.

[6] Guillemin, Fabrice and Leeuwaarden, Johan S.H. van, (2009) Rare event asymptotics for a random walk in the quarter plane, submitted. 
[7] Guillemin, Fabrice and Simonian, Alain, (2007) Asymptotics for random walks in the quarter plane with queueing applications, submitted.

[8] Hunter, Jeffrey J., (1983) Mathematical Techniques of Applied Probability Volume 1: Discrete Time Models: Basic Theory, Acedemic Press, Auckland, New Zealand.

[9] Kobayashi, Masahiro and Miyazawa, Masakiyo, (2011) Tail asymptotics of the stationary distribution of a two dimensional reflecting random walk with unbounded jumps, submitted.

[10] Kobayashi, Masahiro and Miyazawa, Masakiyo, (2012) Revisit to the tail asymptotics of the double QBD process for refinement and complete solutions, submitted.

[11] Kolmogorov, A.N. and Fomin, S.V., (1970) Introductory Real Analysis, Dover Publications, Inc., New York.

[12] Osborne, Anthony D., (1999) Complex Variables and their Applications, Addison Wesley Longman, Essex, England.

[13] Parzen, Emanuel, (1962) Stochastic Processes, Holden-Day, Inc. San Francisco.

[14] Ross, Sheldon M., (2010) Introduction to Probability Models 10th Edition, Academic Press, Los Angeles.

[15] Li, Hui and Zhao, Yiqiang Q. (2001) Tail asymptotics for a generalized twodemand queueing model-a kernel method, Queueing Systems, 69, 77-100. 
[16] Li, Hui and Zhao, Yiqiang Q. (2011) A Kernel Method for Exact Tail Asymptotics-Random Walks in the Quarter Plane, submitted.

[17] Zhao, Yiqiang Q, (2012) Random Walk in the Quarter Plane seminar notes, Carleton University, Ottawa, Canada. 